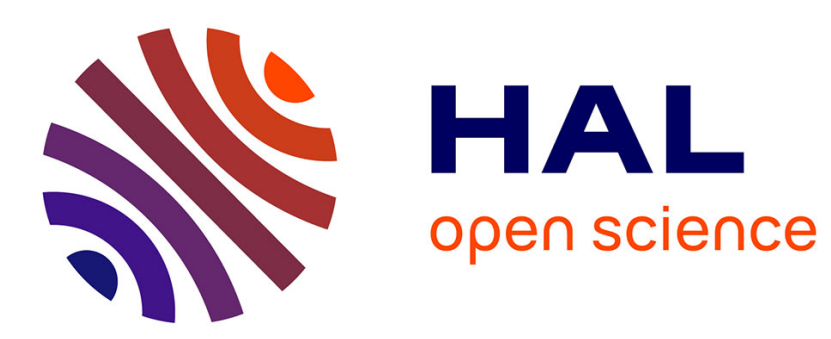

\title{
Aggregation of Bayesian preferences: Unanimity vs Monotonicity
}

Federica Ceron, Vassili Vergopoulos

\section{To cite this version:}

Federica Ceron, Vassili Vergopoulos. Aggregation of Bayesian preferences: Unanimity vs Monotonicity. 2017. halshs-01539444

\section{HAL Id: halshs-01539444 https://shs.hal.science/halshs-01539444}

Submitted on 14 Jun 2017

HAL is a multi-disciplinary open access archive for the deposit and dissemination of scientific research documents, whether they are published or not. The documents may come from teaching and research institutions in France or abroad, or from public or private research centers.
L'archive ouverte pluridisciplinaire HAL, est destinée au dépôt et à la diffusion de documents scientifiques de niveau recherche, publiés ou non, émanant des établissements d'enseignement et de recherche français ou étrangers, des laboratoires publics ou privés. 


\section{Documents de Travail du

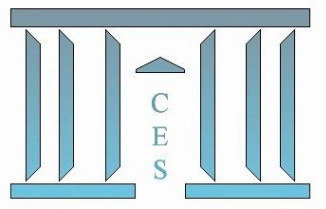

Aggregation of Bayesian preferences:

Unanimity vs Monotonicity

Federica CERon, Vassili Vergopoulos

2017.28

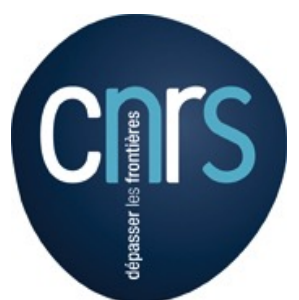

Maison des Sciences Économiques, 106-112 boulevard de L'Hôpital, 75647 Paris Cedex 13 


\title{
Aggregation of Bayesian preferences: Unanimity vs Monotonicity
}

\author{
Federica Ceron* and Vassili Vergopoulos ${ }^{\dagger}$
}

May 2017

\begin{abstract}
This article reconsiders the issue of Bayesian aggregation by pointing at a conflict that may arise between two logically independent dominance criteria, Pareto dominance and statewise dominance, that are commonly imposed on social preferences. We propose a weaker dominance axiom that restricts statewise dominance to Pareto dominant alternatives and Pareto dominance to statewise dominant alternatives. The associated aggregation rule is a convex combination of two components, the first being a weighted sum of the individuals' subjective expected utility (SEU) functional, the second being a social SEU functional, with associated social utility function and social belief. Such representation establishes the existence of a trade off between adherence to the Pareto principle and compliance with statewise dominance. We then investigate what are the consequences of adding to our assumptions either of the two dominance criteria in their full force and obtain that each of them is equivalent to discarding the other, unless there is essentially a common prior probability.
\end{abstract}

Keywords: Pareto dominance, Monotonicity, Preference aggregation, Social choice, Subjective expected utility

JEL classification: D71, D81

\section{Introduction}

Suppose that a group of individuals is asked to express preference judgments over some set of uncertain alternatives and that they all abide to the model of Bayesian rationality. Suppose further that we aim at summarizing the individuals' rankings into a single collective preference judgment. To illustrate, think of a government who aims at implementing an

*Paris School of Economics, Université Paris 1 Panthéon-Sorbonne: federica.ceron@univ-paris1.fr

${ }^{\dagger}$ Paris School of Economics, Université Paris 1 Panthéon-Sorbonne: vassili.vergopoulos@univ-paris1.fr. We are grateful to Jean-Marc Bonnisseau and Jean-Marc Tallon for very useful comments which greatly improved the paper. All errors are our own. 
environmental policy and consults a panel of experts to assess the various risks associated to the available scenarios in order to decide what type of policy to enforce; alternatively, let a social planner be concerned with a tax reform while knowing little about the subjective beliefs of the agents involved; in order to evaluate the welfare of the members of society, the planner asks them to express preference judgments among different specifications of the tax reform and then aggregate these data to define his own ranking. What kind of normative principles should such collective ranking obey? Two natural candidates are the avoidance of arbitrariness - the collective judgment should respect preference judgments over pair of alternatives that the individuals unanimously express - and of inconsistencies - it should be "rational" in the same way that individuals' rankings are. That is, the social preference ranking should be at once Paretian and Bayesian. As we just suggested, these two requirements appear to be quite natural, and there are serious arguments in favour of each: the former desideratum amounts to require the members of the group to be the source of the collective evaluation of states of affairs that emanates from them, or, once the aggregative authority is identified with a social planner who must choose a policy whose outcome is uncertain and affects several individual actors, it demands that the members of society be the source of the decision whose consequences apply to them, a principle grounded in the theory of consumer sovereignty ${ }^{1}$ and tightly related to a welfarist interpretation of social ethics ${ }^{2}$. The second requirement consists in binding the planner's ranking to conform to the same kind of rationality (to the same decision model) as the one of the individual members of society; the rationale behind this can be identified in the principle of coherence, stating that rationality principles are recognized and accepted once and for all, so that they apply both to the individuals' and to the collective evaluations. Here again, attributing the social ranking to (an individual acting as) a social observer rather than conceiving it as the passive result of aggregating individual judgments, seems to strengthen the normative force carried by the coherence principle: the social planner, who adopts a moral rather than personal stand, shall aim at even higher standards of rationality than ordinary individuals. But are these allegedly compelling consistency requisites compatible with each other? This is the so-called issue of consistent Bayesian aggregation ${ }^{3}$. Applying the Pareto principle to the von Neumann-Morgenstern (VNM) apparatus of expected utility for both the individuals and the aggregating authority, Harsanyi (1955) [15] shows that the observer's VNM utility function equals a weighted sum of individual VNM utility functions, and claims to have grounded utilitarianism in a new way ${ }^{4}$. Averaging of individual utilities represents a compromise between personal interests when individuals

\footnotetext{
${ }^{1}$ In social choice theory and welfare economics, consumer sovereignty is the claim that socio-economic decisions are legitimate to the extent only that they originate in the consumers' preferences. Such claim derives its normative stand from the implicit understanding that the satisfaction of one's tastes contributes towards one's welfare or, in a broader sense, that consumer preferences reflect individual welfare.

${ }^{2}$ Welfarism is the (consequentialist) approach to social ethics according to which individual utility values capture all the information on alternatives that may be relevant to the social evaluation.

${ }^{3}$ The term appears in Mongin (1995) [19].

${ }^{4}$ Utilitarianism is the normative view that the rightness of an act depends only on the amount of utility it yields.
} 
disagree, while endorsing the force of their combined authority when they unanimously agree. Yet, the VNM framework presupposes that all individuals face, or agree upon, the same probability distribution over the states of nature that are deemed to be relevant to the collective decision. Hence, divergence of opinion, if any, results solely from different values that the group members attach to the consequences of the chosen action, making such aggregative setting unable to address situations in which not only utility assignments but also factual opinions differ considerably across individuals. To allow individuals to hold different (subjective) beliefs about the likelihood of the states of nature and to have different utilities, the expected utility apparatus needs to be replaced by a framework à la Savage (1954) [24]; under such hypothesis, however, Bayesian aggregation runs into logical difficulties ${ }^{5}$ : it becomes impossible to aggregate individuals' preferences, unless individuals have identical probabilistic beliefs - as in Harsanyi -, or identical taste for outcomes ${ }^{6}$.

To restore the possibility of aggregation, two natural strategies suggest themselves: to relax the Pareto criterion or to depart from the Bayesian rationality requirement, by either allowing the collective preference to embody a different form of rationality than the individuals, or by retaining the coherence between the two, while varying the underlying decision model. There are sound normative arguments in favour of relaxing the Pareto condition, as well as each of Savage's axioms ${ }^{7}$, but we do not intend to pursue either of these strategies here. Rather than rejecting one of the two conditions while retaining the other, we will weaken both Paretianism and Bayesianism at once. The rationale behind this weaker version of double consistency relies on the observation that these two requirements entail an implicit conflict: while Pareto dominance requires each alternative to be evaluated at the collective level solely in terms of individual evaluations, the monotonicity axiom (or statewise dominance), a building block of the formal apparatus of subjective expected utility maximization (henceforth, SEU), requires only the consequences of an act to matter for the collective evaluation. Naturally, under some circumstances, these two instances clash. These circumstances can be identified with binary comparisons of prospects that involve situations of unanimous (individual) preference judgments for a prospect which is statewise dominated according to the collective ranking. Crucially, such a conflicting evaluation can only arise (but needs not) in situations where Pareto domination involves "spurious unanimity", i.e. cases in which the individuals agree on the ranking of two alternatives not by virtue of genuine agreement but because they disagree twice (they hold very different tastes regarding their possible outcomes and very different beliefs on the likelihood of these outcomes) and their conflicting opinions cancel out ${ }^{8}$. If only for the purpose of theoretical experimentation, we want to defuse the outlined conflict while retaining the symmetry

\footnotetext{
${ }^{5}$ For the sake of precision, weaker versions of the ex ante Pareto condition (e.g. weak Pareto or Pareto indifference) yield to dictatorial rules, while the imposition of the strong Pareto condition makes it logically impossible to aggregate individual preferences into a Bayesian collective ranking. See section 4.1 below for a detailed discussion.

${ }^{6}$ Such a result echoes the linear pooling rule in the probability aggregation literature (see for instance Dietrich and List (2016) [11] for an extensive review).

${ }^{7}$ See section 2.1 for a brief review of the existing literature.

${ }^{8}$ The term "spurious unanimity" appears in Mongin (1997) [20].
} 
between individuals and states which is implicit in the standard setting of Bayesian aggregation. Therefore, we propose a weakening of statewise dominance and of the Pareto criteria that restricts statewise dominance to Pareto dominant acts and Pareto dominance to statewise dominant acts. Roughly, we say that a prospect is dominant if it is at the same time Pareto and statewise dominant: agreement across individuals or, symmetrically, across states, is not sufficient to determine the collective preference for some option. As a result, we do not take a stand on which of the two principles should be used a priori to resolve situations of conflict at the expenses of the other: a given collective ranking may be prone to Paretianism in some choice situations while being monotonic in others. Note furthermore that our definition of dominance is implied both by the Pareto condition and by the monotonicity axiom, so if one is willing to accept either of the two, she will be forced to go along with our axiom. The aggregation rule obtained is a convex combination of the two criteria, where the Pareto component is represented by a functional which is ex ante utilitarian in nature and the monotonicity component is represented by means of a social SEU functional, which is ex post utilitarian. The parameter $\gamma$ that determines the weight to be assigned at each of the two consistency requirements is obtained as part of the representation result and may be interpreted as the trade-off between the two ${ }^{9}$; in some sense, by contrasting ex ante and ex post aggregation rules ${ }^{10}$, it may also be viewed as the degree of paternalism of the aggregative authority. We further investigate under what circumstances our aggregation rule is fully Paretian or, symmetrically, fully Bayesian. Abstracting from some minor technicalities, we obtain that full Paretianism (full Bayesianism, respectively) holds when either the parameter $\gamma$ assigns all the weight to the Pareto component of the representation (the monotonicity component, respectively), or there is a common prior, thereby confirming the well-known impossibility results in our setting.

The article is organized as follows: in the next section we provide two examples that motivate the approach adopted, whose main tenets are then briefly outlined. Section 2.1 discusses the related literature, while section 3 describes the technical framework and presents our axioms; the main result, i.e. our aggregation rule, is presented in section 4, while additional results characterizing stronger dominance properties and debating the uniqueness of the representation are contained in sections 4.3 to 4.4. Our conclusions are gathered in section 5. The technical proofs are provided in the Appendix.

\footnotetext{
${ }^{9}$ When $\gamma=1$, we obtain a rule which is utilitarian in spirit, that is, social utility is a convex combination of the individuals' expected utility. When $\gamma=0$, the functional form expresses the case where society disregards individual evaluations and assesses alternatives by taking the expected utility according to its VNM utility function and probabilistic belief.

${ }^{10}$ Ex ante aggregation rules result from applying the Pareto principle to ex ante individual preferences, i.e. to preferences (or, equivalently, SEU functionals) over uncertain prospects; by contrast, ex post aggregation rules restrict the Pareto principle to ex post preferences, that is, preferences relative to consequences only (or, equivalently, to VNM utility indexes). Therefore, ex post rules discard the probability component of individual preferences.
} 


\section{Two examples}

The following two examples will help us illustrating the conflict between Paretian and statewise dominance outlined in the introduction.

Example 1 The duel ${ }^{11}$. Two gentlemen agree to fight a duel, whose result is that one wins and the other loses. The gentlemen have opposite rankings of the three outcomes: (1 wins and 2 loses), (no duel), and (2 wins and 1 loses). Assume that the cardinal rankings of these outcomes are $(1,0,-5)$ for the first gentleman and $(-5,0,1)$ for the second. Moreover, each of them believes, with a probability of at least 85 percent, that he will win the duel. A straightforward adaptation of the Pareto condition implies that society should rank duel (weakly) above no duel, since both prefer it. Yet, for a utilitarian society assigning, say, equal weight to the two gentlemen, the duel leads to one of the gentlemen being severely injured, a net welfare loss of two units of utilities compared to the no duel situation.

Example 2 The choice of education ${ }^{12}$. Consider now a father of two children, who has to finance their higher education. Due to budget constraints, he only has two available strategies: either he funds a three-year BA degree for each child or he funds a eight-year $\mathrm{PhD}$ to only one of them and leaves nothing to the other one. In the latter case, he waits for the next school test to determine who gets the $\mathrm{PhD}$ opportunity. Assume that the welfare of each child is given by the number of years spent in the university, and that the children are pessimistic enough about their own probability to get the best grade, which they both maintain to be of one third. As a result, they unanimously prefer the BA solution. Again however, at the eyes of an utilitarian society assigning equal weights to the children, the $\mathrm{PhD}$ option yields to two more years of university training than the BA option -a net social gain-, no matter which child performs better in the test.

In these examples two seemingly natural evaluation procedures, Pareto dominance and statewise dominance, yield opposite recommendations, leaving the collective ranking of the alternatives involved indeterminate. Since it turns out to be impossible to adhere to the two criteria simultaneously, the question arises as to how to resolve such conflicting situations. When considering the duel example, Paretian logic yields a preference for allowing the duel rather than forbidding it, a solution that might hurt common sense and seems to build a case in favor of restricting the domain of application of the Pareto condition. However, when facing the second example, statewise dominance requires the collective ranking to express strict preference for the $\mathrm{PhD}$ alternative, a solution that may appear as counter-intuitive as the preference for the duel in the previous choice situation. The tenet of our approach is to define a weaker dominance principle to drive collective preferences: an alternative will be said to be dominant if it is at the same time Pareto and statewise dominant; by doing this, we avoid taking a stand on what criterion should

\footnotetext{
${ }^{11}$ The duel story is due to Gilboa, Samet and Schmeidler (2004) [13].

${ }^{12}$ This example is due to Billot and Vergopoulos [4].
} 
the group preferences unilaterally adhere to. Since we do not believe the duel example to be an argument against Pareto nor the education example to be an argument against monotonicity of social preferences, we relax both dominance principles in a symmetric way. As a consequence, a collective ranking may well opt to forbid the duel while financing the BA studies for both children.

\subsubsection{Discussion}

The conflict between unanimity and monotonicity displayed in these examples can be decomposed into two key elements. First, it can only arise in situation of spurious, rather than genuine, unanimity across the group members. Such a phenomenon, first identified by Mongin ( [19], [20], [21]) finds its roots on the fact that, contrary to deterministic or risky choice situations, in uncertain environments two components determine individual preferences $^{13}$ : a taste component (the utility function in the SEU model) and a belief component (the subjective prior probability over the states of the world). It is then possible for individuals to agree on the ranking of two prospects while deeply disagreeing on both factual judgments and utility assessments because these conflicts cancel out. Second, it relies on the utilitarian form of the social utility function, which, as shown by Harsanyi's aggregation theorem, is a consequence of assuming Bayesian rationality for the collective preferences together with the Pareto principle.

Spurious unanimity has been taken as a normative argument against the Pareto principle: since unanimity of judgments can prevail with or without having the individuals agreeing on the reasons for such judgments (that is, on probabilities and utilities), the aggregative authority should take into account the underlying reasons of an individual preference ranking rather than the individual preference ranking by itself; and when those reasons conflict with each other, in the absence of an arbitration principle it is impossible to decide between these opposite arguments, leaving the collective ranking with no motive to commit to the unanimous individual preference nor to the opposite preference. Moreover, under a welfarist interpretation of social ethics ${ }^{14}$, when individual beliefs are contradictory, agreement among individuals cannot be expected to increase the welfare of all members of society, a further argument why such unanimity should not compel society.

The difficulties that enclose the Pareto principle in the context of uncertainty led some authors to restrict the domain of application of the Pareto condition, either by rejecting it at the ex ante stage, i.e. before the resolution of uncertainty, while accepting it ex post,

\footnotetext{
${ }^{13}$ For the sake of precision, it should be noted that deterministic or risky choices are not immune to situations of spurious unanimity, provided that the consequences brought about by an act are aggregates of multiple consequences rather than monolithic. This point has been raised by Mongin (1997) [20], who provides an interesting example to which we refer (p. 14, section 7). It is perhaps interesting to contrast such remark with Hausman and McPherson's well-known comment that it is quite unintuitive to expect people to be better "at forecasting the consequences of lung cancer than the likelihood of getting it" (Hausman and McPherson (1994) [16], p.398).

${ }^{14}$ We recall that an implicit assumption in any approach to social ethics is that utilities and subjective probabilities in Savages theory are meaningful representations of individual tastes and beliefs, rather than pure mathematical constructs.
} 
or by retaining its ex ante formulation but confining it to prospects whose outcome only depends on events to which all individuals assign the same probability. By doing this, any agreement which is rooted in a situation of double disagreement is not treated as genuine agreement anymore (as the ex ante Pareto principle recommends); genuine agreement can only results from unanimity about pure value judgments, emended from probabilistic beliefs, when these differ across individuals.

But while we are sensitive to the case just made against the Pareto principle, we find it troubling to restrict its applicability to pure value judgments or to common-belief prospects, since under some circumstances, e.g. in the above examples, such choice turns out to elect statewise dominance, hence Bayesianism, to be the arbitration principle in case of conflicts between dominance criteria. Abandoning the unrestricted ex ante Pareto principle requires that an individual's prior is to be ignored in making welfare judgments - unless such prior is agreed-upon by all individuals, including the social planner -, a procedure which, beyond being naturally paternalistic, seems to implicitly undermine the validity of Bayesianism as a model of rationality. On the one hand, by accepting to circumscribe the validity of Bayesianism at the individual level, one may wonder why then should the collective preferences be required to be Bayesian; on the other, it seems that retaining the rationality value of SEU stands at odd with the restricted versions of the Pareto principle, at least if one views valuing rational life as recognizing the features which distinguish rational life from other valuable things, specifically the ability of rational creatures to assess reasons and judgments, and to govern their lives in accordance with their assessments ${ }^{15}$.

Another well-recognized objection against Bayesianism for collective preferences concerns the distributional consequences of utilitarianism ${ }^{16}$ : to evaluate social arrangements it may be important to know the exact distribution of individual utilities rather than some sort of mathematical expectation of such utility distribution. We believe that a similar logic is at play in examples 1 and 2, where the monotonic dominance evaluation is driven by the utilitarian form of the collective utility function. Since our weaker dominance principle does not require such an evaluation to be compelling for the observer's preferences, one may view it as allowing fairness considerations to take place.

Arguably, the previous discussion relies on a distinct interpretation of utility and probability as representing pure value and factual judgments, respectively. While such interpretation is widely viewed to be key for any normative discussion of the preference-based approach to social ethics, it is not immune to critics: in real-life one may expect factual and normative considerations to influence both the individuals' evaluations of the conse-

\footnotetext{
${ }^{15}$ Related but more specific arguments against society's interference in individual unanimous evaluations when individuals' factual judgments are incompatible with each other are that (i) prohibiting speculative motives prevents learning in subjective probability models, and (ii) under some circumstances "distorted" beliefs may enhance positive economic outcomes. One such example is provided in Brunnermeier et al. (2014) [5], who consider bubbles caused by heterogeneous beliefs in Akerlof-type lemons models (Akerlof (1970) [1]) and show that they can help overcome market breakdowns induced by the adverse-selection problems.

${ }^{16}$ See Diamond (1967) [10] classic example of a two-person society in which the collective ranking turns out to be indifferent between a lottery involving equal chances for the two agents to receive some positive gain, and another lottery assigning everything to one of the agents with certainty.
} 
quences of certain alternatives and of the likelihood attached to the respective conditioning events. Moreover, Savage theory is perfectly compatible with the view that the utility and probability components of the functional representing preferences may be pure mathematical constructs rationalizing a certain behaviour as defined by the axioms of the theory. According to this view, there is no clear reason why these two elements should be treated differently in the analysis by requiring for instance individuals to be sovereign in normative matters only. It is our conviction that whether one is inclined to embrace the welfarist interpretation of social ethics or rather simply view the collective ranking as the passive result of putting together individual preference judgments, the issue of aggregating group preferences is an interesting one. Importantly, if the welfarist approach seems to strengthen the case in favor of relaxing the Pareto condition, the theoretical aggregation problem may view monotonicity as being a desirable property of the collective ranking which, as opposed to the case of individual preferences, is not however normatively compelling. In short, we remain agnostic as to the normative appeal of Pareto dominance and of monotonicity for the collective ranking. There are serious arguments for and against each of the two. By pointing at a conflict that the two requirements implicitly entail, we ask what is the specific role they play in the context of preference aggregation.

\subsection{Related literature}

Harsanyi's pioneering aggregation theorem (1955) [15] assumes the standard ex ante Pareto principle in the context of VNM expected utility preferences over lotteries, that is prospects depending on agreed-upon probabilities, for both the individuals and the social observer. The derived aggregation rule is a weighted form of utilitarianism. When the VNM framework is replaced by a subjective expected utility (SEU) setting à la Savage and individual beliefs are allowed to be heterogeneous, on the other hand, relatively weak versions of the ex ante Pareto condition (such as weak Pareto or Pareto indifference) yield to dictatorial rules, while the imposition of the strong Pareto condition makes it logically impossible to aggregate individual preferences into a Bayesian collective ranking, unless individuals all have identical tastes - or identical utility functions, up to positive affine transformations. Such negative conclusions have been established both within Savage's axiom set (Hylland and Zeckhauser, 1979 [17]; Mongin, 1995 [19]) and within the Anscombe-Aumann [3] formalization of SEU theory (Mongin, 1998 [21]). More generally, Mongin ( [19], [20], [21]) proves that when individual preferences satisfy some diversity condition, such as the affine independence of the associated utility functions or, symmetrically, of the associated subjective beliefs, then the Pareto condition can only hold if every non-null individual has identical beliefs or identical utility function, respectively. Replacing the assumption of SEU for the collective preferences with suitable monotonicity assumptions with respect to both states and individuals, Mongin and Pivato (2015) [22] strengthen Mongin's negative results. Their analysis is similar to ours in so far the symmetry between Paretian and statewise dominance (and incompatibility thereof) is acknowledged. Chambers and Hayashi (2014) [7] pursue a similar pattern in a framework where there is uncertainty not only as to the true state of the world, but also as to the other agents' preferences. Their 
Pareto condition is then formulated to apply to situations of common knowledge of the appropriate events. These largely negative conclusions established in the field of collective Bayesian decision-making suggest three natural ways out, i.e. abandoning the full force of the Pareto principle, questioning the rationality of Savage's axioms on the collective preference ranking or doing both. The first approach has been adopted by Gilboa, Samet and Schmeidler (2004) [13], who, in the Savage's framework, restrict the domain of applicability of the Pareto principle to "common-belief" prospects, i.e. alternatives whose outcome only depends on events that are consensual in the sense that all agents attach the same probability to them. The aggregation rule obtained recommends using a weighted average of the individuals' probabilities as the observer's beliefs while resorting to the utilitarian social utility function as in Harsanyi's theorem. Investigating the more general setting of Maxmin preferences in the Anscombe-Aumann setting, Qu (2014) [23] obtains the same characterization by restricting the Pareto principle to common-taste acts. Both restrictions of the Pareto principle exclude unanimities that are spurious and they do so by exploiting the rich structure of states and outcomes that are featured by the Savage and AnscombeAumann settings, respectively. These approaches obviously differ from ours since Pareto dominance is relaxed but the collective ranking remains fully monotonic. Among the advocates of relaxing the assumption of Bayesianism at the collective level ${ }^{17}$, Chambers and Hayashi (2006) [6] abandon the requirement of state-independence in a setting à la Savage. Given the well-known relation between monotonicity and state-independence, their paper is maybe the closest to ours. Their aggregation rule, despite the technical differences, echoes ours, when $\gamma$ equals 1. A related result is proven by Mongin (1998) [21] in the Anscombe-Aumann framework; the author also assumes that individual preferences are state-dependent.

Finally, among the writers who relax Bayesianism and Paretianism at once, Danan, Gajdos, Hill, Tallon (2016) [8] relax the completeness property of individual and social preferences by assuming preferences à la Bewley, who allow decision makers to have imprecise probabilistic beliefs in the sense of considering more than one prior as plausible. Yet, they discuss the particular case wherein individual preferences may be complete while those of society are incomplete. In such a context, if individual tastes and beliefs are heterogeneous, the standard Pareto principle is satisfied only if social preferences coincide with those of a particular individual, who then acts as a dictator. Therefore, they show that the assumption that society has a precise belief is not necessary for the difficulties surrounding the issue of Paretian aggregation. In a second result, the Pareto principle is weakened to common-belief options and obtain that the social utility function is utilitarian while the social set of priors is composed by probabilities which are weighted averages of the individual priors. Alon and Gayer (2016) [2] replace Savage's axiom P2 (the sure thing principle) with Maxmin expected utility assumptions for the collective ranking, since they view heterogeneity of individual priors as evidence of the fact that there is ambiguity as

\footnotetext{
${ }^{17}$ Several authors abandoned the hypothesis of Bayesianism at the individual and collective level, i.e. investigated the issue of group preference aggregation under a different decision model. We do not review this literature here.
} 
to what prior should the observer adopt to evaluate a social alternative. They further restrict the Pareto principle to common-belief and common-taste options and obtain an aggregation rule which is very similar to the one proposed by Danan, Gajdos, Hill, Tallon (2016) [8]. Further incomplete social rankings have been obtained by redefining Pareto dominance so as to exclude betting situations (spurious unanimities). Gilboa, Samuleson and Schmeidler (2014) [14] propose a criterion according to which an option $f$ no-betting dominates another option $g$ if $f$ Pareto dominates $g$ and there exists a probability distribution which makes the choice of $f$ individually rational for all agents involved in the sense that all involved individuals' expected utility of $f$ under $p$ is higher than the one of $g$. Brunnermeier, Simsek and Xiong (2014) [5] suggest a belief neutral social welfare criterion that essentially requires an option to be dominant if it is so according to all priors in the convex hull of the individuals.

\section{Framework}

\subsection{Basic framework}

\subsubsection{Uncertain social decision.}

We assume a basic Anscombe-Aumann (1963) framework. Let $X$ be a non-empty set of consequences and $Y=\Delta(X)$ be the set of all lotteries over $X$; that is, distributions over $X$ with finite support. Uncertainty is represented by a finite state space $S$. Let $\mathcal{F}$ be the set of acts; that is, functions from $S$ to $Y$. Since $Y$ is a mixture space, one can define, for any $f, g \in \mathcal{F}$ and for any $\alpha \in[0,1]$, the act $\alpha f+(1-\alpha) g$ in $\mathcal{F}$ which yields $\alpha f(s)+(1-\alpha) g(s) \in Y$ for every state $s \in S$. We slightly abuse notation and denote by $l$ the constant act in $\mathcal{F}$ yielding lottery $l \in Y$ in every state. Finally, for any subset $E \subseteq S$ and any two act $f, g \in \mathcal{F}, f_{E} g$ denotes the element of $\mathcal{F}$ which is equal to $f$ over $E$ and equal to $g$ over $S \backslash E$. If $E=\{s\}$ is a singleton set for some $s \in S$, then we rather use the notation $f_{s} g$ for $f_{E} g$.

\subsubsection{Individuals.}

We assume a society made of a finite number of agents $N=\{1, \ldots, n\}$ with $n \geq 1$. Each agent $i \in N$ in this society is characterized with a preference relation $\succsim_{i}$ over $\mathcal{F}$. As customary, $\sim_{i}$ and $\succ_{i}$ denote its symmetric and asymmetric components.

lottery $l \in Y$ in every state.

\subsubsection{Society.}

A social planner, which we simply refer to as "the society" for convenience, must make decisions. His preferences are given by a binary relation $\succsim_{0}$ on $\mathcal{F}$. Again, $\sim_{0}$ and $\succ_{0}$ denote its symmetric and asymmetric components. The subscript $i=0$ refers to society. 
The set $N \cup\{0\}$ is denoted $N_{*}$. Our goal is to provide an axiomatic representation of social preferences.

\subsection{Assumptions}

SEU individuals: Individual preferences are assumed to abide by the (Anscombe-Aumann) theory of Subjective Expected Utility (SEU). Therefore, for each individual $i \in N$, there exist a probability measure $\lambda_{i}$ on $S$ and a non-constant mixture affine function $u_{i}: Y \rightarrow \mathbb{R}$ such that, for any $f, g \in \mathcal{F}$, we have

$$
f \succsim_{i} g \Longleftrightarrow \mathbb{E}_{\lambda_{i}}\left(u_{i} \circ f\right) \geq \mathbb{E}_{\lambda_{i}}\left(u_{i} \circ g\right) .
$$

Next, we require that, for any finite set of lotteries, the existence of some pair of lotteries that all individual consider as better and worse than any of the lotteries in the set. Such an assumption constitutes a strengthening of a technical condition, ofter referred as minimum agreement on consequences (MAC), which is quite common in the preference aggregation literature and states that there exists two lotteries whose (strict) ranking is commonly agreed by all agents ${ }^{18}$. While we do not believe such strengthening of MAC to be overly restrictive (we may disagree on what is preferable between economic growth and equality, and yet agree that having both outscores having none), we mention that such assumption plays only a technical role in our proof: it is needed to derive the existence of social certainty equivalents without hinging on monotonicity of $\succsim_{0}$ nor on some unanimity (Pareto-like) condition.

C-Agreement: For all finite subset $A$ of $Y$, there exist two lotteries $l, l^{\prime} \in Y$ such that, for all $i \in N$, we have $l \succsim_{i} m \succsim_{i} l^{\prime}$ for all $m \in A$.

As a consequence of c-Agreement, there exist two lotteries $l_{1}, l_{0} \in Y$ such that $l_{1} \succ_{i} l_{0}$ for any $i \in N$. Thus, we can assume without loss of generality that $u_{i}\left(l_{1}\right)=1$ and $u_{i}\left(l_{0}\right)=0$ for any $i \in N$.

\section{Main result}

This section contains the main results of the paper. We first introduce the set of axioms we wish to impose on the social preferences and then present the functional representation to which they are equivalent. Our axioms are weaker than the standard aggregative setting à la Anscombe-Aumann ${ }^{19}$, in that the (Anscombe-Aumann) monotonicity requirement and

\footnotetext{
${ }^{18}$ Formally, MAC postulates the existence of two lotteries $l, m \in Y$ such that for all $i+1, \ldots, n, l \succ_{i} m$. We mention en passant that in the presence of some diversity condition on the individual risk preferences (VNM utility functions) - such as affine independence - MAC becomes an inference rather than a hypothesis, i.e. it holds for free.

${ }^{19}$ That is, SEU rationality together with the (weak) Pareto condition; for a discussion of the latter, see Remark 4.1 and Footnote 20.
} 
the weak Pareto condition are combined in a weaker dominance axiom stating that if an option statewise dominates anther option according to the social conditional preferences and it is preferred to the other by all individuals, then the social unconditional preferences agree with such ranking. Since such weaker dominance principle restricts the applicability of the Pareto condition and of social monotonicity to a particular subsets of act, we further investigate what are the consequences of assuming the unrestricted versions of each of these two conditions in turns (their joint imposition being discarded by the classical impossibility results). Such exercise yields an intuitive characterization of the incompatibility of the two principles in terms of functional representation. We finally show that the representation of social preferences obtained is suitably unique and propose a simple comparative criterion to determine whether a social planner is more prone to monotonicity or Paretianism than another.

\subsection{Axioms}

The first three axioms are the standard Anscombe-Aumann (henceforth, AA) conditions.

(Weak Order) $\succsim_{0}$ is complete and transitive.

(Continuity) For all $f, g, h \in \mathcal{F}$, if $f \succ_{0} g \succ_{0} h$, there exist $\alpha, \beta \in(0,1)$ such that $\alpha f+(1-\alpha) h \succ_{0} g \succ_{0} \beta f+(1-\beta) h$.

(Independence) For all $f, g, h \in \mathcal{F}$ and $\alpha \in(0,1), f \succsim_{0} g$ if and only if $\alpha f+(1-\alpha) h \succsim_{0}$ $\alpha g+(1-\alpha) h$.

Next axiom is novel with respect to the AA framework. It states that for any act $f$, one can always find two lotteries (or equivalently, constant acts) that society values as (weakly) better and worst than $f$. As for the technical assumption of c-agreement, this condition is needed to derive the existence of certainty equivalents for the social preferences without hinging on monotonicity. It is easy to see that such condition is a minor consequence of the standard AA monotonicity.

(Boundedness) For all $f \in \mathcal{F}$, there exist $l, m \in Y$ such that $l \succsim_{0} f \succsim_{0} m$.

The following axiom is the key assumption to our approach; it states that if all individuals unanimously prefer (weakly) an option $f$ to another option $g$, and the former option statewise dominates the latter from the social viewpoint, then society should also prefer $f$ to $g$. It is easily observed that such condition represents at once a weakening of statewise dominance and of the Pareto criteria; it restricts statewise dominance to Pareto dominant acts and Pareto dominance to statewise dominant acts. Observe that the standard Pareto condition or, symmetrically, AA monotonicity each implies Weak dominance, which is therefore hardly refutable by whoever is willing to accept either of the two. 
(Weak Dominance) For all $f, g \in \mathcal{F}$, if $f \succsim_{i} g$ for any $i \in N$ and $f(s) \succsim_{0} g(s)$ for any $s \in S$, then $f \succsim_{0} g$.

Remark: Note that the unanimity condition used in the above axiom is the weak version of the Pareto principle ${ }^{20}$; we mentioned in the introduction that, when individual tastes and beliefs are heterogeneous, the joint imposition of weak Pareto and SEU assumptions on the individuals' and on the collective ranking delivers dictatorial rules rather than a logical impossibility. Here, the choice of weak Pareto is motivated by our intention of guaranteeing a symmetric treatment of the two dominance conditions, for to assume a strong version of statewise dominance is of little meaning.

Finally, a non-triviality assumption is needed.

(Non-triviality) We have $l_{1} \succ_{0} l_{0}$.

Such condition is stronger than the usual AA non-triviality axiom, since it requires strict preference to hold with respect to two specific rather than arbitrary lotteries. However, it is not necessary to the proof: it only allows a convenient normalization of the collective VNM index which simplifies the presentation of the result.

\subsection{Result}

In order to introduce our main result, the following definition is needed. We say that a function $u_{0}: Y \rightarrow \mathbb{R}$ is normalized if $u_{0}\left(l_{1}\right)=1$ and $u_{0}\left(l_{0}\right)=0$.

Theorem 1 Assume SEU individuals and c-Agreement. Then, society's preferences $\succsim_{0}$ satisfy Weak Order, Continuity, Independence, Boundedness, Weak Dominance and Nontriviality if and only if there exist non-negative real numbers $\left(\alpha_{1}, \ldots, \alpha_{n}\right)$ with $\alpha_{1}+\ldots+$ $\alpha_{n}=1$, a real number $\gamma \in[0,1]$, a probability measure $\lambda_{0}$ on $S$ and a normalized mixture affine function $u_{0}: Y \rightarrow \mathbb{R}$ satisfying the following:

(Th1.1) For any $l \in Y, \gamma \cdot u_{0}(l)=\gamma \cdot\left(\alpha_{1} \cdot u_{1}(l)+\ldots+\alpha_{n} \cdot u_{n}(l)\right)$

(Th1.2) For any $f, g \in \mathcal{F}, f \succsim_{0} g \Longleftrightarrow V_{0}(f) \geq V_{0}(g)$, where, for any $h \in \mathcal{F}$,

$$
V_{0}(h)=\gamma \cdot \sum_{i \in N} \alpha_{i} \cdot \mathbb{E}_{\lambda_{i}}\left(u_{i} \circ h\right)+(1-\gamma) \cdot \mathbb{E}_{\lambda_{0}}\left(u_{0} \circ h\right) .
$$

Example 3 Consider again examples 1 and 2. The collective evaluation ranks no duel above duel whenever $\gamma<\frac{20}{21}$, while it ranks the BA option higher than the Phd one whenever

\footnotetext{
${ }^{20}$ The weak Pareto principle states that if all agents weakly prefer one option to another, so does the collective ranking, while the strong (sometimes called strict) Pareto condition requires that if all agents weakly prefer one prospect to another and there exists at least one agent whose preference is strict, society has also a strict preference for the first option.
} 
$\gamma>\frac{3}{4}$. Hence, and contrary to a unilateral commitment to one of the dominance criteria, there is an interval of values for the parameter $\gamma$ (namely $\gamma \in\left(\frac{3}{4}, \frac{20}{21}\right)$ ) that allows society to forbid the duel among the two gentlemen while financing BA studies for both children.

Theorem 1 posits an aggregation rule which is a convex combination of two components, the first being a weighted sum of the individuals' SEU functional, i.e. of the expected values of the prospect computed according to the individual utility function and prior belief, the second being the social SEU functional, with associated social utility function and social belief. Since the first term trivially satisfies the (ex ante) Pareto condition (but does not entail a separation of social beliefs and tastes), while the second one is clearly monotonic, theorem 1 establishes the existence of a trade off between adherence to the Pareto principle and compliance with statewise dominance. While the Pareto component of the representation is necessarily ex ante utilitarian, the monotonicity component has an ex post utilitarian form whenever the parameter $\gamma$, determining the weight to be assigned to Pareto dominance (and, consequently, to statewise dominance), is strictly positive. Hence, if Pareto is at all to matter, utilitarianism follows. On the other hand, when $\gamma=0$, the functional form expresses the case where society disregards individual evaluations and assesses alternatives by taking the expected utility according to its (unrestricted) VNM utility function and probabilistic belief. By the same token, when $\gamma=1$, only ex ante individual evaluations matter for the social evaluations, so there is no room for a social VNM index. A formal analysis of these limit cases is provided below and shows, roughly, that under heterogeneity of individual beliefs they are equivalent to assume the full force of either of the dominance criteria at the expenses of the other.

A final remark concerns the existence of a social belief $\lambda_{0}$ and of a social utility function $u_{0}$ that need not be related to, nor stem from, individual beliefs and utility assignments respectively. The above representation involves a SEU social planner who treats her own preferences as the ones of any of the individual members of society and then applies the Pareto principle. Indeed, by letting $\beta_{i}=\gamma \cdot \alpha_{i}$ for all $i \in \mathcal{N}$ and $\beta_{0}=1-\gamma$, representation 2 can be written as follows:

$$
V_{0}(h)=\sum_{j \in N_{*}} \beta_{j} \cdot \mathbb{E}_{\lambda_{j}}\left(u_{j} \circ h\right)
$$

Such feature is in line with the spirit of our Weak dominance principle; in some sense, individual evaluations alone or, symmetrically, social considerations alone are not sufficient to determine the final deliberation, which therefore turns out to be a compromise between the two. The Pareto principle is a reason to restrict social preferences and social preferences provide a reason to contrast unanimous judgments.

\subsection{Pareto Dominance and Statewise Dominance}

In this section we investigate the implications of strengthening our assumptions by assuming the full force of the Pareto principle or of monotonicity on the representation provided in theorem 1. Put it differently, we ask how the aggregation rule changes once either of 
the dominance criteria is added to our axiom set. As it is customary in the literature, we focus on situations where individuals tastes, as represented by their utility functions, are sufficiently diverse. An established formal rendering of such diversity consists in assuming the existence of pair of lotteries that "separate" each agent's preferences in the sense that every individual in society but one agent is indifferent between the two. Such assumption, stated below under the label of risk diversity and assumed throughout this section, is wellknown to be equivalent to the individuals' utility functions being linearly independent ${ }^{21}$. Interestingly, we obtain that full Paretianism (full Bayesianism, respectively) holds when either the parameter $\gamma$ assigns all the weight to the Pareto component of the representation (the monotonicity component, respectively), or there is a common prior, thereby confirming the well-known negative conclusions on the impossibility of Bayesian aggregation in our setting. Given such clear-cut characterization, it is then possible to compare classes of preferences as defined in theorem 1 according to their disposition to Paretianism (or, dually, to Bayesianism). Loosely speaking, we say that a social planner is more prone to Paretianism than another one if, whenever the first social planner respects unanimity of individual preferences, so does the latter. Dually, we say that a planner is more prone to statewise dominance than another if, whenever she complies with the axiom of monotonicity, so does the latter. Not surprisingly, the two dominance criteria being incompatible, a planner is more prone to Paretianism than another if and only if she is less prone to statewise dominance. Furthermore, it suffices to compare the magnitude of the parameter $\gamma$ in order to identify the social planner's disposition toward Paretianism.

Finally, given the well-known relationship between monotonicity and state-independence of preferences, one may expect social preferences as defined by theorem 1 to be in general state-dependent. We confirm such intuition below by showing that social preferences are state-independent if and only if monotonicity holds.

We are now ready to state such results formally. For the rest of this subsection, we assume that society's preferences $\succsim_{0}$ satisfy Weak Order, Continuity, Independence, Boundedness, Weak Dominance and Non-triviality. Let the structure $\left(\gamma,\left(\alpha_{i}\right)_{i \in N}, u_{0}, \lambda_{0}\right)$ provide a representation of $\succsim_{0}$ as in Theorem 1 . We will furthermore assume the diversity condition mentioned at the beginning of this section:

Risk Diversity: For any $i \in N$, there exist $l, m \in Y$ such that $l \succ_{i} m$ and $l \sim_{j} m$ for all $j \in N \backslash\{i\}$.

To state our first proposition formally, some additional definitions are needed. We first remind the notions of Pareto, and statewise dominance.

(Pareto Dominance) For all $f, g \in \mathcal{F}$, if $f \succsim_{i} g$ for any $i \in N$, then $f \succsim_{0} g$.

(Statewise Dominance) For all $f, g \in \mathcal{F}$, if $f(s) \succsim_{0} g(s)$ for any $s \in S$, then $f \succsim_{0} g$.

\footnotetext{
${ }^{21}$ Somewhat surprisingly, this diversity condition also implies that individuals cannot be in full disagreement either, for it implies the property of minimal agreement on consequences, that we already discussed in section 3.2 above.
} 
Remark: Our Weak dominance axiom used the conjunction of the antecedents of Pareto dominance and Statewise dominance as antecedent while delivering the same consequent. It is perhaps clearer now in what sense Weak dominance is implied by each dominance criterion separately.

We further introduce the notion of state-independence of preferences, which is based on the following definition of null state:

A state $s \in S$ is said to be null if any two acts being equal to each other on $S \backslash\{s\}$ are indifferent for $\succsim_{0}$. We can now define state-independence of preferences.

(State Independence) For any $s \in S, f \in \mathcal{F}$, and $l, m \in Y$, if $l_{s} f \succ_{0} m_{s} f$, then $l_{t} f \succ_{0} m_{t} f$ for any nonnull state $t \in S^{22}$.

Next, we define an individual to be null if her preferences are irrelevant to society, and call a prior probability common if it is shared by every non-null individual. Such common prior probability may or not coincide with the social prior probability. Furthermore, we use the definition of null agents to distinguish between two notions of convex combination of individual utilities: the classic definition involving non-negative coefficients, and the particular case where individual weights are bound to be strictly positive whenever an agent is non-null.

We say that that a given individual $i \in N$ is null if there exist $f, g \in \mathcal{F}$ such that $f \succ_{i} g$, $f \sim_{j} g$ for any $j \in N \backslash\{i\}, f(s) \sim_{0} g(s)$ for any $s \in S$, and yet $f \sim_{0} g$.

We say that the social prior is essentially a common prior if, for any non-null individual $i \in N$, we have $\lambda_{i}=\lambda_{0}$. We say that there is essentially a common prior if there exist a probability measure $\lambda$ on $S$ such that, for any non-null individual $i \in N$, we have $\lambda_{i}=\lambda$.

We say that $u_{0}$ is a convex combination of individual utilities if there exist non-negative coefficients $\left\{\beta_{1}, \ldots, \beta_{n}\right\}$ summing to 1 such that $u_{0}(l)=\sum_{i \in N} \beta_{i} \cdot u_{i}(l)$ for any $l \in Y$. If additionally we have that $i \in N$ is non-null if and only if $\beta_{i}>0$, we say that the convex combination is adapted.

Proposition 1 Assume SEU individuals, c-Agreement and Risk Diversity. Let the structure $\left\{\gamma,\left(\alpha_{i}\right)_{i \in N}, u_{0}, \lambda_{0}\right\}$ provide a representation of $\succsim_{0}$ as in Theorem 1 . Then, the following equivalences hold:

(P1.1) Pareto Dominance holds iff $\gamma=1$, or $\lambda_{0}$ is essentially a common prior, and $u_{0}$ is an adapted convex combination of individual utilities.

(P1.2) Statewise Dominance holds iff $\gamma=0$, or there is essentially a common prior.

(P1.3) Statewise Dominance holds iff State Independence holds.

\footnotetext{
${ }^{22}$ This axiom appears, among others, in Fishburn (1970, p. 177) [12] and Kreps (1988, p. 109) [18].
} 
The first part of Proposition 1 shows that Pareto dominance holds when either the monotonicity component (the social SEU functional) disappears from the representation - yielding a pure ex ante utilitarian aggregation rule -, or the social VNM index is utilitarian and the social prior is essentially common, i.e. every non-null individual and the social planner have the same prior. In some sense, either the social observer is itself "null" and social preferences are not SEU, or her prior probability coincides with the one of any other non-null individual and a SEU representation follows even though monotonicity has not been assumed. Note that the notion of essentially common social prior generalizes the one of probability dictatorship ${ }^{23}$, so that the case where $\gamma \neq 1$ essentially strengthens (but nevertheless does not generalize ${ }^{24}$ ) the classical result of Bayesian aggregation ${ }^{25}$ : if an agent's utility assignment is to be given any weight, the social planner should adopt her same beliefs; reciprocally, and unless the social planner agrees with an agent's beliefs, the agent's utility plays no role in the aggregation rule.

The second part of proposition 1 posits the equivalence between statewise dominance and the irrelevance of the preferences of the individuals, unless they essentially agree on the prior probability. In the latter case, social preferences are represented by a SEU functional with associated utilitarian VNM index and probability given by the convex combination (with weight $\gamma$ ) of such common prior and the social belief $\lambda_{0}$, as if the planner were compromising between her beliefs and those shared by the individuals. Such result is perhaps the most surprising, given that social preferences may ignore or overturn the ones of the individuals. Yet adherence to statewise dominance means in some sense that the preferences of the observer are not to be ignored when making social decisions which, in the presence of sufficient individual heterogeneity, commonly results in dictatorial solutions; therefore, we see (P1.1) and (P1.2) as two sides of the same coin.

Finally, the last equivalence formally establishes that social preference are state-independent whenever they satisfy statewise dominance.

We turn now to the comparative notion discussed at the beginning of this section, i.e. we ask whether it is possible to identify social planners who are more prone to Pareto or statewise dominance than others. As mentioned earlier, we will focus on social observers whose preferences are strongly comparable, in the sense that they assign the same set of individual weights, and they have same social belief and VNM index. We will also discard the case where there is essentially a common prior.

Formally, consider now two preference relations over $\mathcal{F}$ given by $\succsim_{0}$ and $\succsim_{0}^{\prime}$. We say that $\succsim_{0}$ is more prone to Pareto Dominance than $\succsim_{0}^{\prime}$ if for any $f, g \in \mathcal{F}$ such that $f \succsim_{i} g$ for all $i \in N, f \succsim_{0}^{\prime} g$ implies $f \succsim_{0} g$. We say that $\succsim_{0}$ is more prone to Statewise Dominance

\footnotetext{
${ }^{23}$ Formally, an individual $i \in \mathcal{N}$ is a probability dictator if $\lambda_{0}=\lambda_{i}$; this definition is due to Mongin (1998) [21].

${ }^{24}$ This is because while the aggregation rule involves a generalization of probability dictatorship, it does so by displaying a stronger form of convexity of individual utilities for the social VNM utility.

${ }^{25}$ By classical result we mean the fact that the joint imposition of SEU assumptions on the individuals and the social observer together with (weak) Pareto dominance and linear independence of individual utilities yield to probability dictatorship; see for example lemma 5 and proposition 9 in Mongin (1998) [21].
} 
than $\succsim_{0}^{\prime}$ if for any $f, g \in \mathcal{F}$ such that $f(s) \succsim_{0} g(s)$ and $f(s) \succsim_{0}^{\prime} g(s)$ for all $s \in S, f \succsim_{0}^{\prime} g$ implies $f \succsim_{0} g$.

Proposition 2 Assume SEU individuals, c-Agreement and Risk Diversity. Consider two binary relations $\succsim_{0}$ and $\succsim_{0}^{\prime}$ on $\mathcal{F}$. Assume that $\succsim_{0}$ and $\succsim_{0}^{\prime}$ define the same set of null individuals, and that there is essentially no common prior. Let the structures $\left\{\gamma,\left(\alpha_{i}\right)_{i \in N}, u_{0}, \lambda_{0}\right\}$ and $\left\{\delta,\left(\alpha_{i}\right)_{i \in N}, u_{0}, \lambda_{0}\right\}$ provide two representations as in Theorem 1 for $\succsim_{0}$ and $\succsim_{0}^{\prime}$ respectively. Then, the following are equivalent:

(P2.1) $\succsim_{0}$ is more prone to Pareto Dominance than $\succsim_{0}^{\prime}$,

(P2.2) $\succsim_{0}$ is less prone to Statewise Dominance than $\succsim_{0}^{\prime}$,

$(\mathrm{P} 2.3) \gamma \geq \delta$.

\subsection{Uniqueness of the representation}

In this section we study the uniqueness properties of the representation provided in theorem 1. We will need to distinguish two fundamental situations, depending on whether an essentially common prior exists or not. In the former case the representation turns out to be essentially unique, meaning that the relevant parameters - i.e. those parameters that do not necessarily cancel out from the aggregation rule - are unique. Clearly, the remaining parameters may well take arbitrary values, but since any such value delivers exactly the same final representation of social preferences, there is no serious loss. When there is an essentially common prior, on the other hand, social preferences admits a unique SEU representation which, however, given the parsimony of its parameters, cannot be associated to a unique structure $\left\{\gamma,\left(\alpha_{i}\right)_{i \in N}, u_{0}, \lambda_{0}\right\}$ as provided by theorem 1 . Any of such structures representing the same social preferences $\succsim_{0}$ is in fact admissible, provided that a few constraints linking the parameters of the two representations are satisfied. This is not surprising, for reducing to a SEU representation involves an important loss of information with respect to the representation provided in theorem 1. Such loss is not immune to critiques, for in many contexts very different arrangements may turn out (doubtfully) to be formally equivalent, but it is not an uncommon feature of functional aggregators.

Proposition 3 Assume SEU individuals, c-Agreement, Risk Diversity and that there is essentially no common prior. Let the structures $\left\{\gamma,\left(\alpha_{i}\right)_{i \in N}, u_{0}, \lambda_{0}\right\}$ and $\left\{\delta,\left(\beta_{i}\right)_{i \in N}, v_{0}, \mu_{0}\right\}$ provide two representations of $\succsim_{0}$ as in Theorem 1. Then, we always have $\delta=\gamma$ and $v_{0}=u_{0}$. Moreover, the following hold:

(P3.1) If $\gamma=\delta=0$, then $\mu_{0}=\lambda_{0}$.

(P3.2) If $\gamma=\delta=1$, then $\beta_{i}=\alpha_{i}$ for any $i \in N$.

(P3.3) If $\gamma=\delta \in(0,1)$, then $\mu_{0}=\lambda_{0}$ and $\beta_{i}=\alpha_{i}$ for any $i \in N$.

Proposition 4 Assume SEU individuals and c-Agreement. Assume that there is essentially a common prior denoted by $\lambda$. Then, society's preferences $\succsim_{0}$ satisfy Weak Order, 
Continuity, Independence, Boundedness, Weak Dominance and Non-triviality if and only if there exist a normalized mixture affine function $v_{0}: Y \rightarrow \mathbb{R}$ and a probability measure $\mu_{0}$ on $S$ such that for any $f, g \in \mathcal{F}$,

$$
f \succsim_{0} g \Longleftrightarrow \mathbb{E}_{\mu_{0}}\left(v_{0} \circ f\right) \geq \mathbb{E}_{\mu_{0}}\left(v_{0} \circ g\right) \text {. }
$$

Moreover, $v_{0}$ and $\mu_{0}$ are unique. Finally, for any structure $\left\{\gamma,\left(\alpha_{i}\right)_{i \in N}, u_{0}, \lambda_{0}\right\}$ providing a representation of $\succsim_{0}$ as in Theorem 1, we have:

(P4.1) $v_{0}=u_{0}$,

(P4.2) If $\gamma>0$, then $v_{0}=\sum_{i \in N} \alpha_{i} \cdot u_{i}$,

(P4.3) $\mu_{0}=\gamma \cdot \lambda+(1-\gamma) \cdot \lambda_{0}$.

\section{Conclusions}

This article reconsiders the issue of Bayesian aggregation by pointing at a conflict that may arise between two logically independent dominance criteria, Pareto dominance and statewise dominance, that are commonly imposed on social preferences. We propose a weaker dominance axiom that restricts statewise dominance to Pareto dominant alternatives and Pareto dominance to statewise dominant alternatives. In this way we maintain the symmetry between the relevance of individuals and of states of nature to the social evaluation which is implicit in the standard setting while avoiding to take a stand as per what criterion should be adopted at the expenses of the other. The associated aggregation rule is a convex combination of two components, the first being a weighted sum of the individuals' SEU functional, the second being a social SEU functional, with associated social utility function and social belief. Since the first term trivially satisfies the (ex ante) Pareto condition, while the second one is clearly monotonic, such representation establishes the existence of a trade off between adherence to the Pareto principle and compliance with statewise dominance. We then investigate what are the consequences of adding to our assumptions either of the two dominance criteria in their full force and obtain that each of them is equivalent to discarding the other dominance principle, unless there is essentially a common prior probability.

\section{Appendix: proofs of all results}

\subsection{Four useful lemmata}

Lemma 1 Consider an integer $P \geq 1$, and a mapping $\varphi_{p}: \mathcal{F} \rightarrow \mathbb{R}$ for any $p \in[0 \ldots P]$. Suppose that each $\varphi_{p}$ is mixture affine; that is, for any $f, g \in \mathcal{F}$ and any $\alpha \in[0,1]$, $\varphi_{p}(\alpha f+(1-\alpha) g)=\alpha \varphi_{p}(f)+(1-\alpha) \varphi_{p}(g)$. Moreover, suppose that the $\varphi_{p}$ are related by 
a Pareto condition; that is, for any $f, g \in \mathcal{F}$, if $\varphi_{p}(f) \geq \varphi_{p}(g)$ for any $p \in[1 \ldots P]$, then $\varphi_{0}(f) \geq \varphi_{0}(g)$.

Then, there exist non-negative numbers $\alpha_{1}, \ldots, \alpha_{P}$ and a real number $\beta$ such that, for any $f \in \mathcal{F}$, we have

$$
\varphi_{0}(f)=\sum_{p=1}^{P} \alpha_{p} \cdot \varphi_{p}(f)+\beta .
$$

Proof Define a mapping $\Phi: \mathcal{F} \rightarrow \mathbb{R}^{P+1}$ by, for any $f \in \mathcal{F}, \Phi(f)=\left(\varphi_{0}(f), \ldots, \varphi_{P}(f)\right)$. Let $K \subseteq \mathbb{R}^{P+1}$ denote the range of $\Phi$. Since $\varphi_{p}$ is mixture affine for any $p \in[0 \ldots P]$, the subset $K$ must be convex. Moreover, since the $\varphi_{p}$ are related by a Pareto condition, we can invoke Proposition 1 in De Meyer and Mongin (1995) [9] and finally obtain $\alpha_{1}, \ldots, \alpha_{P}$ and $\beta$ as in Equation (5).

Lemma 2 Let the structure $\left\{\gamma,\left(\alpha_{i}\right)_{i \in N}, u_{0}, \lambda_{0}\right\}$ provide a representation of $\succsim_{0}$ as in Theorem 1 with $\gamma>0$. Then, for any $i \in N, i$ is null if and only if $\alpha_{i}=0$.

Proof First, suppose that $i$ is null. Then, there exist $f, g \in \mathcal{F}$ such that: (a) $f \succ_{i} g$, (b) $f \sim_{j} g$ for any $j \in N \backslash\{i\},\left(\right.$ c) $f(s) \sim_{0} g(s)$ for any $s \in S$, and yet (d) $f \sim_{0} g$. Since the structure $\left\{\gamma,\left(\alpha_{i}\right)_{i \in N}, u_{0}, \lambda_{0}\right\}$ provide a representation of $\succsim_{0}$ as in Theorem 1 , and by Conditions (b), (c) and (d),

$$
0=V_{0}(f)-V_{0}(g)=\gamma \cdot \alpha_{i} \cdot\left[V_{i}(f)-V_{i}(g)\right]
$$

By Condition (a), we have $V_{i}(f)-V_{i}(g)>0$. Since $\gamma>0$ by assumption, we must have $\alpha_{i}=0$. Now, suppose that $\alpha_{i}=0$. By Risk Diversity, there are $l, m \in Y$ such that $l \succ_{i} m$ and $l \sim_{j} m$ for any $j \in N \backslash\{i\}$. Let $s \in S$ be a state such that $\lambda_{i}(s)>0$. Define $f, g \in \mathcal{F}$ by $g\left(s^{\prime}\right)=m$ for any $s^{\prime} \in S$, and $f\left(s^{\prime}\right)=l$ if $s^{\prime}=s$ and $f\left(s^{\prime}\right)=m$ otherwise. By construction, we have $f \sim_{j} g$ for any $j \in N \backslash\{i\}$, and $f \sim_{i} g$. Moreover, since $\gamma>0$ and $\alpha_{i}=0$, we have $u_{0}=\sum_{j \neq i} \alpha_{j} u_{j}$ by (Th1.1). So $u_{0}(f(s))=u_{0}(g(s))$ and, therefore, $f(s) \sim_{0} g(s)$ for any $s \in S$. But then we must also have $\mathbb{E}_{\lambda_{0}}\left(u_{0} \circ f\right)=\mathbb{E}_{\lambda_{0}}\left(u_{0} \circ g\right)$. Finally, by (Th1.2), we also have for any $h \in \mathcal{F}$

$$
V_{0}(h)=\gamma \cdot \sum_{j \in N \backslash\{i\}} \alpha_{j} \cdot V_{j}(h)+(1-\gamma) \cdot \mathbb{E}_{\lambda_{0}}\left(u_{0} \circ h\right)
$$

Therefore, $V_{0}(f)=V_{0}(g)$, and $f \sim_{0} g$. This shows that $i$ is null.

Lemma 3 Assume Risk Diversity. For any function $\psi: N \times S \rightarrow \mathbb{R}$ such that, for any $f \in \mathcal{F}$,

$$
\sum_{i \in N, s \in S} \psi(i, s) \cdot u_{i}(f(s))=0
$$


we have $\psi(i, s)=0$ for any $i \in N$ and $s \in S$.

Proof Fix $i \in N$ and $s \in S$. We construct $f, g \in \mathcal{F}$ such that $f(t) \sim_{j} g(t)$ for any $(j, t) \in N \times S$ with $(j, t) \neq(i, s)$, and $f(s) \succ_{i} g(s)$. By Risk Diversity, there exist $l, m \in Y$ such that $l \succ_{i} m$ and $l \sim_{j} m$. for any $j \in N \backslash\{i\}$. Let $g \in \mathcal{F}$ be such that $g(t)=m$ for any $t \in S$. Let $f \in \mathcal{F}$ be such that $f(t)=l$ if $t=s$ and $f(t)=m$ otherwise. These two acts satisfy the conditions above. Then, by Equation (6), we have

$$
0=\sum_{j \in N, t \in S} \psi(j, t) \cdot u_{j}(f(t))-\sum_{j \in N, t \in S} \psi(j, t) \cdot u_{j}(g(t))=\psi(i, s) \cdot\left[u_{i}(f(s))-u_{i}(g(s))\right] .
$$

But by construction we have $u_{i}(f(s))>u_{i}(g(s))$. So it must be that $\psi(i, s)=0$. This holds for any $i \in N$ and $s \in S$.

We say that $\succsim_{0}$ satisfies $S T P$ if for any $E \subseteq S$ and any $f, g, h, k \in \mathcal{F}$, we have $f_{E} h \succsim_{0} g_{E} h$ iff $f_{E} k \succsim_{0} g_{E} k$.

Lemma 4 If $\succsim_{0}$ satisfies completeness, transitivity, STP and State Independence, then it satisfies Statewise Dominance.

Proof Suppose that $\succsim_{0}$ satisfies STP and State Independence. We have:

Claim 1: For any $s \in S, f, g \in \mathcal{F}$, and $l, m \in Y$, if $l_{s} f \succ_{0} m_{s} f$, then $l_{t} g \succ_{0} m_{t} g$ for any nonnull state $t \in S$.

Indeed, suppose $l_{s} f \succ_{0} m_{s} f$ for some $s \in S, f \in \mathcal{F}$ and $l, m \in Y$. Let $t \in S$ be a nonnull state. Then, by State Independence, $l_{t} f \succ_{0} m_{t} f$. Finally, by STP, we obtain $l_{t} g \succ_{0} m_{t} g$.

Claim 2: For any $s \in S, f \in \mathcal{F}$, and $l, m \in Y$, if $l \succsim_{0} m$, then $l_{s} f \succsim_{0} m_{s} f$.

Let $S^{*}:=\left\{s_{1}, \ldots s_{N}\right\}$ be the set of nonnull states in $S$. Consider $s \in S, f \in \mathcal{F}$, and $l, m \in Y$ such that $l_{s} f \succ_{0} m_{s} f$. We will show that $l \succ_{0} m$. By Claim 1, we obtain:

$$
l_{t} g \succ_{0} m_{t} g \quad \text { for any } t \in S^{*} \text { and any } g \in \mathcal{F} \text {. }
$$

In particular, for $t=s_{1}$ and $g=l$, we get $l \succ_{0} m_{s_{1}} l$.

Now, note that $m_{s_{1}} l=l_{s_{2}}\left(m_{s_{1}} l\right)$. By applying (7) to $t=s_{2}$ and $g=m_{s_{1}} l$, we get $m_{s_{1}} l=l_{s_{2}}\left(m_{s_{1}} l\right) \succ_{0} m_{s_{2}}\left(m_{s_{1}} l\right)=m_{\left\{s_{1}, s_{2}\right\}} l$. By the conclusion of the previous paragraph, $l \succ_{0} m_{\left\{s_{1}, s_{2}\right\}} l$.

By repeating iteratively this process, we obtain $l \succ_{0} m_{S^{*}} l$. Now, let $S \backslash S^{*}=\left\{t_{1}, \ldots, t_{M}\right\}$. Note that $m_{S^{*}} l$ and $m_{S^{*} \cup\left\{t_{1}\right\}} l$ are equal to each other except on the null state $t_{1}$. So $l \succ_{0} m_{S^{*}} l \sim_{0} m_{S^{*} \cup\left\{t_{1}\right\}} l$. Moreover, $m_{S^{*} \cup\left\{t_{1}\right\}} l$ and $m_{S^{*} \cup\left\{t_{1}, t_{2}\right\}} l$ are equal to each other except on the null state $t_{2}$. So $m_{S^{*} \cup\left\{t_{1}\right\}} l \sim_{0} m_{S^{*} \cup\left\{t_{1}, t_{2}\right\}} l$ and, therefore, $l \succ_{0} m_{S^{*} \cup\left\{t_{1}, t_{2}\right\}} l$. By repeating iteratively this process, we finally obtain $l \succ_{0} m_{S} l=m$, as desired. 
Claim 3: $\succsim_{0}$ satisfies Statewise Dominance.

Let $S=\left\{s_{1}, \ldots, s_{N}\right\}$. Let $f, g \in \mathcal{F}$ be such that $f(s) \succsim_{0} g(s)$ for any $s \in S$. Define $f_{0}=f$ and, for any $n \in[1 \ldots N], f_{n}=g\left(s_{n}\right)_{s_{n}} f_{n-1}$. For any $n \in[1 \ldots N]$, we clearly have $f_{n-1}\left(s_{n}\right)=f\left(s_{n}\right) \succsim_{0} g\left(s_{n}\right)$ so, by Claim $2, f_{n-1}\left(s_{n}\right)_{s_{n}} f_{n-1} \succsim_{0} g\left(s_{n}\right)_{s_{n}} f_{n-1}$; that is, $f_{n-1} \succsim_{0} f_{n}$. Then, $f_{0} \succsim_{0} f_{N}$; that is, $f \succsim_{0} g$.

Claim 3 completes the proof of the lemma.

\subsection{Proof of Theorem 1}

The necessity of Weak Order, Continuity and Independence is straightforward. So we only prove the necessity of Weak Dominance, Boundedness and Non-triviality. To do so, assume a representation as in Theorem 1 . Since $u_{0}$ is normalized, we have $u_{0}\left(l_{1}\right)>u_{0}\left(l_{0}\right)$. Moreover, by confronting (Th1.1) and (Th1.2), we see that $V_{0}\left(l^{\prime}\right)=u_{0}\left(l^{\prime}\right)$ for any $l^{\prime} \in Y$. Thus, $V_{0}\left(l_{1}\right)>V_{0}\left(l_{0}\right)$. But since $V_{0}$ represents social preferences, we finally obtain $l_{1} \succ_{0} l_{0}$. To show Weak Dominance, assume that $f, g \in \mathcal{F}$ satisfy $f \succsim_{i} g$ for any $i \in N$ and $f(s) \succsim_{0} g(s)$ for any $s \in S$. Then, we have $\mathbb{E}_{\lambda_{i}}\left(u_{i} \circ f\right) \geq \mathbb{E}_{\lambda_{i}}\left(u_{i} \circ g\right)$ for any $i \in N$ and $V_{0}(f(s)) \geq V_{0}(g(s))$ for any $s \in S$. Since we have proved that $u_{0}$ is the restriction of $V_{0}$ to lotteries, we have $u_{0}(f(s)) \geq u_{0}(g(s))$ and, therefore, $\mathbb{E}_{\lambda_{0}}\left(u_{0} \circ f\right) \geq \mathbb{E}_{\lambda_{0}}\left(u_{0} \circ g\right)$. By equation (2), this is sufficient to obtain $V_{0}(f) \geq V_{0}(g)$ and, finally, $f \succsim_{0} g$. Hence Weak Dominance. Finally, to show Boundedness, consider an arbitrary act $f \in \mathcal{F}$. If $\gamma=0$, then $\succsim_{0}$ satisfies Satatewise Dominance. Moreover, the range of $f$ is finite. So there exist $l, m \in Y$ such that $l \succsim_{0} f(s) \succsim_{0} m$. Then, by Statewise Dominance, we have $l \succsim_{0} f \succsim_{0} m$. If $\gamma>0$, then by (Th1.1) we have $u_{0}=\sum_{i \in N} \alpha_{i} u_{i}$. Since the range of $f$ is finite, we can apply c-Agreement and obtain $l, m \in Y$ such that $l \succsim_{i} f(s) \succsim_{i} m$ for any $s \in S$ and $i \in N$. Since individual preferences satisfy Statewise Dominance, we obtain $l \succsim_{i} f \succsim_{i} m$ for any $i \in N$. Moreover, we have $u_{i}(l) \geq u_{i}(f(s)) \geq u_{i}(m)$ for any $s \in S$ and $i \in N$. Since $u_{0}=\sum_{i \in N} \alpha_{i} u_{i}$, we obtain $u_{0}(l) \geq u_{0}(f(s)) \geq u_{0}(m)$ for any $s \in S$. Therefore, $l \succsim_{0} f(s) \succsim_{0} m$ for any $s \in S$. Then, we can apply Weak Dominance since we have already proved that it is necessary, and get $l \succsim_{0} f \succsim_{0} m$.

From now on, we assume that social preferences satisfy Weak Order, Continuity, Independence, Boundedness, Weak Dominance and Non-triviality. Observe that Weak order, Continuity and Independence imply that the restriction of $\succsim_{0}$ to lotteries satisfies the von Neumann and Morgenstern axioms. Then, by their theorem, there exists a mixture affine function $u_{0}: Y \rightarrow \mathbb{R}$ that provides a representation for the restriction of $\succsim_{0}$ to lotteries. Moreover, by Non-triviality, we must have $u_{0}\left(l_{1}\right)>u_{0}\left(l_{0}\right)$. By applying positive affine transformations if necessary, we may suppose without loss of generality that $u_{0}\left(l_{1}\right)=1$ and $u_{0}\left(l_{0}\right)=0$. Thus, $u_{0}$ is normalized.

Now, to construct a functional $V_{0}$ providing a representation for $\succsim_{0}$, we first show that 
each act $f \in \mathcal{F}$ has a certainty equivalent for $\succsim_{0}$. That is, for any $f \in \mathcal{F}$, there exists $l \in Y$ such that $f \sim_{0} l$. Fix $f \in \mathcal{F}$. By Boundedness, there are $l, m \in Y$ such that $l \succsim_{0} f \succsim_{0} m$. Then, the construction of a certainty equivalent follows from standard arguments, which we only briefly sketch. First, if $f \sim_{0} l$ or $f \sim_{0} m$, we are done. So we assume without loss of generality that $l \succ_{0} f \succ_{0} \mathrm{~m}$. Then, the sets

$$
\left\{\alpha \in[0,1], \quad \alpha l+(1-\alpha) m \succ_{0} f\right\} \quad \text { and } \quad\left\{\alpha \in[0,1], \quad f \succ_{0} \alpha l+(1-\alpha) m\right\}
$$

are of the form $(\alpha, 1]$ and $[0, \alpha)$ respectively, for some $\alpha \in(0,1)$. Then, the lottery $p \in Y$ defined by $p=\alpha l+(1-\alpha) m$ satisfies $f \sim_{0} p$.

Next, define the function $V_{0}: \mathcal{F} \rightarrow \mathbb{R}$ by, for any $f \in \mathcal{F}$ :

$$
V_{0}(f)=u_{0}(l)
$$

where $l$ is a certainty equivalent associated to $f$. Clearly, $V_{0}(f)$ is well-defined, independent of the choice of a specific certainty equivalent since $u_{0}$ represents the restriction of $\succsim_{0}$ to $Y$. Moreover, $V_{0}$ represents social preferences; that is, for any $f, g \in \mathcal{F}, f \succsim_{0} g$ if and only if $V_{0}(f) \geq V_{0}(g)$. Last, it is simple to use the axiom of Independence to see that $V_{0}$ is mixture-linear on $\mathcal{F}$.

Note that the functional $V_{i}$ is mixture affine for any $i \in N$. So is the functional $f \rightarrow$ $u_{0}(f(s))$ for any $s \in S$. Let $k$ be the cardinalty of $S$. Since Weak Dominance holds, Lemma 1 provides non-negative numbers $\left(\sigma_{j}\right)_{j=1}^{n+k} \in \mathbb{R}^{n+k}$ and a real number $\mu \in \mathbb{R}$ such that, for any $f \in \mathcal{F}$,

$$
V_{0}(f)=\sum_{i \in N} \sigma_{i} \cdot V_{i}(f)+\sum_{s \in S} \sigma_{s} \cdot u_{0}(f(s))+\mu .
$$

By assumption, we have $V_{i}\left(l_{1}\right)=u_{i}\left(l_{1}\right)=1$ and $V_{i}\left(l_{0}\right)=u_{i}\left(l_{0}\right)=0$ for any $i \in N$. Meanwhile, by construction, we have $V_{0}\left(l_{1}\right)=u_{0}\left(l_{1}\right)=1$ and $V_{0}\left(l_{0}\right)=u_{0}\left(l_{0}\right)=0$. Then, it must necessarily be that $\sigma:=\sum_{j \in N \cup S} \sigma_{j}=1$ and $\mu=0$. Next, define $\gamma=\sum_{i \in N} \sigma_{i}$. There are now different cases:

Case 1: $\gamma=0$. Then, set $\lambda_{0}(s)=\sigma_{s}$ for $s \in S$. This defines a probability measure $\lambda_{0}$ on $S$, and we obtain $V_{0}(f)=\mathbb{E}_{\lambda_{0}}\left(u_{0} \circ f\right)$. Hence the representation of Theorem 1 .

Case 2: $\gamma=1$. Then, set $\alpha_{i}=\sigma_{i}$ for $i \in N$. These numbers $\alpha_{i}$ are non-negative and sum up to 1 . We obtain $V_{0}(f)=\sum_{i \in N} \alpha_{i} \cdot \mathbb{E}_{\lambda_{i}}\left(u_{i} \circ f\right)$. Hence the representation of Theorem 1 .

Case 3: $\gamma \in(0,1)$. Then, set $\lambda_{0}(s)=\sigma_{s} /(1-\gamma)$ for $s \in S$ and $\alpha_{i}=\sigma_{i} / \gamma$ for $i \in N$ to obtain the representation of Theorem 1 . 
Thus, in all three cases, we have

$$
V_{0}(h)=\gamma \cdot \sum_{i \in N} \alpha_{i} \cdot \mathbb{E}_{\lambda_{i}}\left(u_{i} \circ h\right)+(1-\gamma) \cdot \mathbb{E}_{\lambda_{0}}\left(u_{0} \circ h\right)
$$

Given that $u_{0}$ is the restriction of $V_{0}$ to lotteries, applying the latter equation to the case of a constant act establishes (Th1.2).

\subsection{Proof of Proposition 1}

Claim 1: If $\succsim_{0}$ satisfies Pareto Dominance and if $\gamma \in(0,1)$, then the social prior is essentially a common prior, and $u_{0}$ is an adapted convex combination of $\left\{u_{i}, i \in N\right\}$.

Since $\gamma>0$, we already know from (Th1.1) that $u_{0}$ is a convex combination of $\left\{u_{i}, i \in N\right\}$ with coefficients given by $\left\{\alpha_{i}, i \in N\right\}$. By Lemma 2, this convex combination is adapted. Moreover, note that $V_{i}$ is mixture affine for any $i \in N_{*}$. Since Pareto Dominance holds, Lemma 1 provides non-negative numbers $\beta_{i}, i \in N$, and $\mu \in \mathbb{R}$ such that, for any $f \in \mathcal{F}$,

$$
V_{0}(f)=\sum_{i \in N} \beta_{i} \cdot V_{i}(f)+\mu .
$$

If we apply this to $f=l_{1}, l_{0}$, we obtain $\mu=0$ and $\sum_{i \in N} \beta_{i}=1$ since $u_{0}$ is normalized and since it is the restriction of $V_{0}$ to lotteries. But since $\gamma>0$, by (Th1.1) we have $u_{0}=\sum_{i \in N} \alpha_{i} \cdot u_{i}$. So we must have

$$
\sum_{i \in N} \alpha_{i} \cdot u_{i}=u_{0}=\sum_{i \in N} \beta_{i} \cdot u_{i}
$$

Fix $i \in N$ and let $l, m \in Y$ be as in Risk Diversity. Then, since $l \sim_{j} m$ for any $j \in N \backslash\{i\}$

$$
\begin{aligned}
0 & =\sum_{j \in N}\left(\alpha_{j}-\beta_{j}\right) \cdot u_{j}(l)-\sum_{i \in N}\left(\alpha_{j}-\beta_{j}\right) \cdot u_{j}(m) \\
& =\left(\alpha_{i}-\beta_{i}\right) \cdot\left(u_{i}(l)-u_{i}(m)\right)
\end{aligned}
$$

Since by construction $l \succ_{i} m$, we must have $\alpha_{i}=\beta_{i}$. Therefore, for any $f \in \mathcal{F}$, we obtain

$$
V_{0}(f)=\sum_{i \in N} \alpha_{i} \cdot V_{i}(f)
$$

On the one hand, by Equation (2) in (Th1.2), we have for any act $f \in \mathcal{F}$,

$$
V_{0}(f)=\sum_{i \in N, s \in S}\left[\gamma \cdot \alpha_{i} \cdot \lambda_{i}(s)+(1-\gamma) \cdot \alpha_{i} \cdot \lambda_{0}(s)\right] \cdot u_{i}(f(s)) .
$$


On the other hand, by Equation (9)

$$
V_{0}(f)=\sum_{i \in N, s \in S} \alpha_{i} \cdot \lambda_{i}(s) \cdot u_{i}(f(s))
$$

Then, define $\psi(i, s)=\left[\gamma \alpha_{i} \lambda_{i}(s)+(1-\gamma) \alpha_{i} \lambda_{0}(s)\right]-\alpha_{i} \lambda_{i}(s)$ for any $i \in N$ and $s \in S$. By confronting Equations (10) and (11) and applying Lemma 3, we obtain:

$$
\gamma \cdot \alpha_{i} \cdot \lambda_{i}(s)+(1-\gamma) \cdot \alpha_{i} \cdot \lambda_{0}(s)=\alpha_{i} \cdot \lambda_{i}(s)
$$

Rearranging Equation (12), and using $\gamma<1$, we obtain that $\lambda_{i}=\lambda_{0}$ for any $i \in N$ such that $\alpha_{i}>0$. But by Lemma 2, $i \in N$ is non-null if and only if $\alpha_{i}>0$. So for any non-null individual $i \in N$, we have $\lambda_{i}=\lambda_{0}$. Hence, the social prior is essentially a common prior.

Claim 2: If $\succsim_{0}$ satisfies Pareto Dominance and if $\gamma=0$, then the social prior is essentially a common prior, and $u_{0}$ is an adapted convex combination of $\left\{u_{i}, i \in N\right\}$

Note that $V_{i}$ is mixture affine for any $i \in N_{*}$. Since Pareto Dominance holds, Lemma 1 provides non-negative numbers $\beta_{i}, i \in N$, and $\mu \in \mathbb{R}$ such that, for any $f \in \mathcal{F}$,

$$
V_{0}(f)=\sum_{i \in N} \beta_{i} \cdot V_{i}(f)+\mu .
$$

If we apply this to $f=l_{1}, l_{0}$, we obtain $\mu=0$ and $\sum_{i \in N} \beta_{i}=1$ since $u_{0}$ is normalized and since it is the restriction of $V_{0}$ to lotteries. Thus, we obtain, for any $f \in \mathcal{F}$,

$$
V_{0}(f)=\sum_{i \in N, s \in S} \beta_{i} \cdot \lambda_{i}(s) \cdot u_{i}(f(s))
$$

In particular, we have $u_{0}=\sum_{i \in N} \beta_{i} \cdot u_{i}$. This shows that $u_{0}$ is a convex combination of individual utilities. Moreover, Equation (13) provides another representation of $\succsim_{0}$ as in Theorem 1. By applying Lemma 2 to this other representation, $i \in N$ is non-null if and only if $\beta_{i}>0$. So the convex combination is adapted. Since $\gamma=0$, Equation (2) in (Th1.2) gives, for any $f \in \mathcal{F}$,

$$
V_{0}(f)=\sum_{i \in N, s \in S} \beta_{i} \cdot \lambda_{0}(s) \cdot u_{i}(f(s)) .
$$

Then, define $\psi(i, s)=\beta_{i} \lambda_{i}(s)-\beta_{i} \lambda_{0}(s)$ for any $i \in N$ and $s \in S$. By confronting Equations (13) and (14) and applying Lemma 3, we obtain for any $i \in N$ and $s \in S$ :

$$
\beta_{i} \cdot \lambda_{i}(s)=\beta_{i} \cdot \lambda_{0}(s)
$$

So if $\beta_{i}>0$, then $\lambda_{i}=\lambda_{0}$. Then, if $i \in N$ is non-null, we have $\beta_{i}>0$ and therefore $\lambda_{i}=\lambda_{0}$. The social prior is essentially a common prior.

Combining Claims 1 and 2, we obtain that if Pareto Dominance holds, then either $\gamma=1$, 
or the social prior is essentially a common prior, both when $\gamma>0$ (Claim 1) and when $\gamma=0$ (Claim 2). Moreover, $u_{0}$ is always an adapted convex combination of $\left\{u_{i}, i \in N\right\}$. Reciprocally, assume that $\gamma=1$, or the social prior is essentially a common prior and $u_{0}$ is an adapted convex combination of $\left\{u_{i}, i \in N\right\}$. We show Pareto Dominance. If $\gamma=1$, this is trivial given Equation (2). So we assume that $\gamma<1$.

Case 1: $\gamma>0$. Then, by (Th1.2) we have $u_{0}=\sum_{i \in N} \alpha_{i} u_{i}$. Let $I$ stand for the set of $i \in N$ that are non-null. Then, by Lemma $2, I=\left\{i \in N, \alpha_{i}>0\right\}$. Since the social prior is essentially a common prior, we have that if $i \in I$, then $\lambda_{i}=\lambda_{0}$. Then, Equation (2) gives for any $f \in \mathcal{F}$,

$$
\begin{aligned}
V_{0}(f) & =\gamma \cdot \sum_{i \in N} \alpha_{i} \cdot \mathbb{E}_{\lambda_{i}}\left(u_{i} \circ f\right)+(1-\gamma) \cdot \sum_{i \in N} \alpha_{i} \cdot \mathbb{E}_{\lambda_{0}}\left(u_{i} \circ f\right) \\
& =\gamma \cdot \sum_{i \in N} \alpha_{i} \cdot \mathbb{E}_{\lambda_{i}}\left(u_{i} \circ f\right)+(1-\gamma) \cdot \sum_{i \in I} \alpha_{i} \cdot \mathbb{E}_{\lambda_{i}}\left(u_{i} \circ f\right) \\
& =\gamma \cdot \sum_{i \in N} \alpha_{i} \cdot \mathbb{E}_{\lambda_{i}}\left(u_{i} \circ f\right)+(1-\gamma) \cdot \sum_{i \in N} \alpha_{i} \cdot \mathbb{E}_{\lambda_{i}}\left(u_{i} \circ f\right) \\
& =\sum_{i \in N} \alpha_{i} \cdot \mathbb{E}_{\lambda_{i}}\left(u_{i} \circ f\right) .
\end{aligned}
$$

Then, it is straightforward to see that Pareto Dominance holds.

Case 2: $\gamma=0$. Let $I$ stand for the set of $i \in N$ that are non-null. By assumption, there exist non-negative coefficients $\left(\beta_{1}, \ldots, \beta_{n}\right)$ summing to 1 such that $u_{0}=\sum_{i \in N} \beta_{i} u_{i}$ and $I=\left\{i \in N, \beta_{i}>0\right\}$. Proceeding as in Case 1, we obtain $V_{0}(f)=\sum_{i \in N} \beta_{i} \cdot \mathbb{E}_{\lambda_{i}}\left(u_{i} \circ f\right)$ for any $f \in \mathcal{F}$. Hence Pareto Dominance. This completes the proof of (P1.1).

Claim 3: If $\succsim_{0}$ satisfies Statewise Dominance and if $\gamma>0$, then there is essentially a common prior.

For any $s \in S$, the mapping $f \rightarrow u_{0}(f(s))$ is mixture affine; so is $V_{0}$. Since Statewise Dominance holds, Lemma 1 gives the existence of non-negative numbers $\mu_{0}(s), s \in S$, and $\mu \in \mathbb{R}$ such that, for any $f \in \mathcal{F}$,

$$
V_{0}(f)=\sum_{s \in S} \mu_{0}(s) \cdot u_{0}(f(s))+\mu .
$$

If we apply this to $f=l_{1}, l_{0}$, we obtain $\mu=0$ and $\sum_{s \in S} \mu_{0}(s)=1$ since $u_{0}$ is normalized and since it is the restriction of $V_{0}$ to lotteries. Thus, $\mu_{0}$ defines a probability measure on $S$. Moreover, since $\gamma>0$, by (Th1.1) we have $u_{0}=\sum_{i \in N} \alpha_{i} u_{i}$. Then, for any $f \in \mathcal{F}$,

$$
V_{0}(f)=\sum_{i \in N, s \in S} \alpha_{i} \cdot \mu_{0}(s) \cdot u_{i}(f(s))
$$


On the other hand, by Equation (2), we have for any $f \in \mathcal{F}$,

$$
V_{0}(f)=\sum_{i \in N, s \in S}\left[\gamma \cdot \alpha_{i} \cdot \lambda_{i}(s)+(1-\gamma) \cdot \alpha_{i} \cdot \lambda_{0}(s)\right] \cdot u_{i}(f(s)) . .
$$

Then, define $\psi(i, s)=\left[\gamma \alpha_{i} \lambda_{i}(s)+(1-\gamma) \alpha_{i} \lambda_{0}(s)\right]-\alpha_{i} \mu_{0}(s)$ for any $i \in N$ and $s \in S$. By confronting Equations (16) and (17) and applying Lemma 3, we obtain:

$$
\gamma \cdot \alpha_{i} \cdot \lambda_{i}(s)+(1-\gamma) \cdot \alpha_{i} \cdot \lambda_{0}(s)=\alpha_{i} \cdot \mu_{0}(s) .
$$

Therefore, for any $i \in N$ such that $\alpha_{i}>0$, we have $\gamma \cdot \lambda_{i}+(1-\gamma) \cdot \lambda_{0}=\mu_{0}$. Since $\gamma>0$, this shows that $\lambda_{i}$ is independent of $i$ provided $i$ satisfies $\alpha_{i}>0$. Let $\lambda$ denote this common measure. To conclude, if $i$ is non-null, then by Lemma 2, $\alpha_{i}>0$ and therefore $\lambda_{i}=\lambda$. So there is essentially a common prior.

Claim 3 shows if Statewise Dominance holds, then either $\gamma=0$, or there is essentially a common prior. Reciprocally, if $\gamma=0$, it is straightfroward to show Statewise Dominance given Equation (2). So we assume that $\gamma>0$, and that there is essentially a common prior. By (Th1.1), we have $u_{0}=\sum_{i \in N} \alpha_{i} u_{i}$. Let $\lambda$ be the measure on $S$ such that, for any non-null $i \in N, \lambda_{i}=\lambda$. Note also that, by Lemma $2, i \in N$ is non-null if and only if $\alpha_{i}>0$. Let $I=\left\{i \in N, \alpha_{i}>0\right\}$. For any $f \in \mathcal{F}$, we have:

$$
\begin{aligned}
V_{0}(f) & =\gamma \cdot \sum_{i \in I} \alpha_{i} \cdot \mathbb{E}_{\lambda}\left(u_{i} \circ f\right)+(1-\gamma) \cdot \mathbb{E}_{\lambda_{0}}\left(u_{0} \circ f\right) \\
& =\gamma \cdot \mathbb{E}_{\lambda}\left(\sum_{i \in I} \alpha_{i} \cdot u_{i} \circ f\right)+(1-\gamma) \cdot \mathbb{E}_{\lambda_{0}}\left(u_{0} \circ f\right) \\
& =\gamma \cdot \mathbb{E}_{\lambda}\left(u_{0} \circ f\right)+(1-\gamma) \cdot \mathbb{E}_{\lambda_{0}}\left(u_{0} \circ f\right)
\end{aligned}
$$

Then, $\succsim_{0}$ has an SEU representation where a utility function is given by $u_{0}$ and the prior is given by $\gamma \cdot \lambda+(1-\gamma) \cdot \lambda_{0}$. It becomes straightforward to see that Statewise Dominance holds.

Claim 4: Assume that $\succsim_{0}$ has an SEU representation. Then $\succsim_{0}$ satisfies State Independence.

Let $\mu$ be the prior on $S$ and $v$ be a utility function on $Y$ providing an SEU representation. Let $s \in S, f \in \mathcal{F}$, and $l, m \in Y$ be such that $l_{s} f \succ_{0} m_{s} f$. Then, by the assumed SEU representation, we have $\mu(s) \cdot\{v(l)-v(m)\}>0$. So it must be the case that $v(l)-v(m)>0$. Consider now any nonnull state $t \in S$. We have $\mu(t)>0$ and therefore $\mu(t) \cdot\{v(l)-v(m)\}>0$. But then still by the assumed SEU representation we obtain $l_{t} f \succ_{0} m_{t} f$.

Finally, we show that Statewise Dominance and State Independence are equivalent. First, assume State Independence. Given the representation (2), it is clear that $\succsim_{0}$ satisfies STP. Then, Lemma 4 implies that $\succsim_{0}$ satisfies Statewise Dominance. Now, assume Statewise 
Dominance. By (P1.2), either $\gamma=0$, or there is essentially a common prior denote by $\lambda$. In the first case, $\succsim_{0}$ has an SEU representation with respect to $u_{0}$ and $\lambda_{0}$. In the second case, as shown above, it has an SEU representation with respect to $u_{0}$ and $\gamma \cdot \lambda+(1-\gamma) \cdot \lambda_{0}$. In both cases, it has an SEU representation. Then, by Claim 4, it satisfies State Independence.

\subsection{Proof of Proposition 2}

Let $V_{0}$ and $W_{0}$ be the functionals defined respectively by the structures $\left\{\gamma,\left(\alpha_{i}\right)_{i \in N}, u_{0}, \lambda_{0}\right\}$ and $\left\{\delta,\left(\alpha_{i}\right)_{i \in N}, u_{0}, \lambda_{0}\right\}$ as in equation (2). First, we show the equivalence between (P2.1) and (P2.2).

Assume that $\succsim_{0}$ is more prone to Pareto Dominance that $\succsim_{0}^{\prime}$. Then, $f \succsim_{i} g$ for all $i \in N$ and $f \succsim_{0}^{\prime} g$ imply $f \succsim_{0} g$ for any $f, g \in \mathcal{F}$. Since the functionals $V_{i}$, for $i \in N, V_{0}$ and $W_{0}$ are all mixture affine, Lemma 1 and normalization provide non-negative numbers $\beta_{i}$, for all $i \in N$, and $\beta_{0}$ summing to 1 such that for any $f \in \mathcal{F}$,

$$
V_{0}(f)=\sum_{i \in N} \beta_{i} \cdot V_{i}(f)+\beta_{0} \cdot W_{0}(f) .
$$

Moreover, note if $\delta=0$, then we have $\gamma \geq \delta$. So we can suppose that $\delta>0$. Then, $u_{0}=\sum_{i \in N} \alpha_{i} u_{i}$. Therefore, for any $f \in \mathcal{F}$,

$$
W_{0}(f)=\delta \cdot \sum_{i \in N} \alpha_{i} \cdot \mathbb{E}_{\lambda_{i}}\left(u_{i} \circ f\right)+(1-\delta) \cdot \sum_{i \in N} \alpha_{i} \cdot \mathbb{E}_{\lambda_{0}}\left(u_{i} \circ h\right) .
$$

Combining Equations (19) and (20), we obtain for any $f \in \mathcal{F}$,

$$
V_{0}(f)=\sum_{i \in N, s \in S}\left[\beta_{i} \lambda_{i}(s)+\beta_{0} \delta \alpha_{i} \lambda_{i}(s)+\beta_{0}(1-\delta) \alpha_{i} \lambda_{0}(s)\right] \cdot u_{i}(f(s)) .
$$

On the other hand, by Equation $(2)$ and $u_{0}=\sum_{i \in N} \alpha_{i} u_{i}$, we also have for any $f \in \mathcal{F}$,

$$
V_{0}(f)=\sum_{i \in N, s \in S}\left[\gamma \alpha_{i} \lambda_{i}(s)+(1-\gamma) \alpha_{i} \lambda_{0}(s)\right] \cdot u_{i}(f(s))
$$

Then, define $\psi(i, s)=\left[\beta_{i} \lambda_{i}(s)+\beta_{0} \delta \alpha_{i} \lambda_{i}(s)+\beta_{0}(1-\delta) \alpha_{i} \lambda_{0}(s)\right]-\left[\gamma \alpha_{i} \lambda_{i}(s)+(1-\gamma) \alpha_{i} \lambda_{0}(s)\right]$ for any $i \in N$ and $s \in S$. By confronting Equations (21) and (22) and applying Lemma 3, we obtain for any $i \in N$ and $s \in S$,

$$
\beta_{i} \lambda_{i}(s)+\beta_{0} \delta \alpha_{i} \lambda_{i}(s)+\beta_{0}(1-\delta) \alpha_{i} \lambda_{0}(s)=\gamma \alpha_{i} \lambda_{i}(s)+(1-\gamma) \alpha_{i} \lambda_{0}(s) .
$$

By summing these equalities for $s \in S$, we obtain $\beta_{i}=\left(1-\beta_{0}\right) \alpha_{i}$. Reinjecting this in Equation (23), we obtain for any $i \in N$ such that $\alpha_{i}>0$ and $s \in S$,

$$
\left(1-\beta_{0}\right) \lambda_{i}(s)+\beta_{0} \delta \lambda_{i}(s)+\beta_{0}(1-\delta) \lambda_{0}(s)=\gamma \lambda_{i}(s)+(1-\gamma) \lambda_{0}(s) .
$$


Now, suppose that $1-\beta_{0}+\beta_{0} \delta-\gamma \neq 0$. Then, for any non-null individual $i \in N$, we have $\alpha_{i}>0$ by Lemma 2. Then, since $1-\beta_{0}+\beta_{0} \delta-\gamma \neq 0$, Equation (23) shows that $\lambda_{i}$ is independent of $i$. In other words, there is essentially a common prior, which contradicts our assumptions. Therefore, we must have $1-\gamma=\beta_{0}(1-\delta)$. Then, $1-\gamma \leq 1-\delta$ and finally $\gamma \geq \delta$.

Assume now that $\gamma \geq \delta$. If $\delta=1$, then $\gamma=\delta=1$. Furthermore, we have $V_{0}=W_{0}$. So the two binary relations must agree with each other. Thus, $\succsim_{0}$ is more prone to Pareto Dominance that $\succsim_{0}^{\prime}$. If $\delta<1$, then let $\beta_{0}$ be such that $1-\gamma=\beta_{0}(1-\delta)$, and for all $i \in N$ set $\beta_{i}=\alpha_{i}\left(1-\beta_{0}\right)$. It is then easy to see that Equation (19) holds. Hence, $\succsim_{0}$ is more prone to Pareto Dominance that $\succsim_{0}^{\prime}$.

Now, we show the equivalence between (P2.2) and (P2.3). Assume that $\succsim_{0}$ is less prone to Statewis Dominance that $\succsim_{0}^{\prime}$. Then, since the utility function $u_{0}$ is the same for $\succsim_{0}$ and $\succsim_{0}^{\prime}$, we have: $f(s) \succsim_{0} g(s)$ for all $i \in N$ and $f \succsim_{0} g$ imply $f \succsim_{0}^{\prime} g$ for any $f, g \in \mathcal{F}$. Since the functionals $f \rightarrow u_{0}(f(s))$, for $s \in S, V_{0}$ and $W_{0}$ are all mixture affine, Lemma 1 and normalization provide non-negative numbers $\mu(s)$, for all $s \in S$, and $\mu_{0}$ summing to 1 such that for any $f \in \mathcal{F}$,

$$
W_{0}(f)=\sum_{s \in S} \mu(s) \cdot u_{0}(f(s))+\mu_{0} \cdot V_{0}(f)
$$

Moreover, note if $\delta=0$, then we have $\gamma \geq \delta$. So we can suppose that $\delta>0$. Then, $u_{0}=\sum_{i \in N} \alpha_{i} u_{i}$. Therefore, for any $f \in \mathcal{F}$,

$$
V_{0}(f)=\gamma \cdot \sum_{i \in N} \alpha_{i} \cdot \mathbb{E}_{\lambda_{i}}\left(u_{i} \circ f\right)+(1-\gamma) \cdot \sum_{i \in N} \alpha_{i} \cdot \mathbb{E}_{\lambda_{0}}\left(u_{i} \circ h\right) .
$$

Combining Equations (25) and (26), we obtain for any $f \in \mathcal{F}$,

$$
W_{0}(f)=\sum_{i \in N, s \in S}\left[\alpha_{i} \mu(s)+\mu_{0} \gamma \alpha_{i} \lambda_{i}(s)+\mu_{0}(1-\gamma) \alpha_{i} \lambda_{0}(s)\right] \cdot u_{i}(f(s))
$$

On the other hand, by Equation $(2)$ and $u_{0}=\sum_{i \in N} \alpha_{i} u_{i}$, we also have for any $f \in \mathcal{F}$,

$$
W_{0}(f)=\sum_{i \in N, s \in S}\left[\delta \alpha_{i} \lambda_{i}(s)+(1-\delta) \alpha_{i} \lambda_{0}(s)\right] \cdot u_{i}(f(s))
$$

Then, define $\psi(i, s)=\left[\alpha_{i} \mu(s)+\mu_{0} \gamma \alpha_{i} \lambda_{i}(s)+\mu_{0}(1-\gamma) \alpha_{i} \lambda_{0}(s)\right]-\left[\delta \alpha_{i} \lambda_{i}(s)+(1-\delta) \alpha_{i} \lambda_{0}(s)\right]$ for any $i \in N$ and $s \in S$. By confronting Equations (27) and (28) and applying Lemma 3, we obtain for any $i \in N$ and $s \in S$,

$$
\alpha_{i} \mu(s)+\mu_{0} \gamma \alpha_{i} \lambda_{i}(s)+\mu_{0}(1-\gamma) \alpha_{i} \lambda_{0}(s)=\delta \alpha_{i} \lambda_{i}(s)+(1-\delta) \alpha_{i} \lambda_{0}(s) .
$$

Suppose that $\delta \neq \mu_{0} \gamma$. For any non-null individual $i \in N$, we have $\alpha_{i}>0$ by Lemma 2 
and, therefore,

$$
\mu+\mu_{0} \gamma \lambda_{i}+\mu_{0}(1-\gamma) \lambda_{0}=\delta \lambda_{i}+(1-\delta) \lambda_{0} .
$$

Equation (30) shows that $\lambda_{i}$ is independent of $i$. In other words, there is essentially a common prior, which contradicts our assumptions. Therefore, we must have $\delta=\mu_{0} \gamma \leq \gamma$. Now, suppose $\delta \leq \gamma$. If $\gamma=0$, then $\delta=\gamma=0$. Furthermore, we have $V_{0}=W_{0}$. So the two binary relations must agree with each other. Thus, $\succsim_{0}$ is less prone to Statewise Dominance that $\succsim_{0}^{\prime}$. If $\gamma>0$, then let $\mu_{0}$ be such that $\delta=\mu_{0} \gamma$. For any $s \in S$, set $\mu(s)=\left(1-\mu_{0}\right) \lambda_{0}(s)$. It is then easy to see that Equation (25) holds. Hence, $\succsim_{0}$ is less prone to Statewise Dominance that $\succsim_{0}^{\prime}$.

\subsection{Proof of Proposition 3}

Suppose first $\gamma=0$. Then, by Equation (2), $\succsim_{0}$ must satisfy Statewise Dominance. But then, by Proposition 1, either $\delta=0$, or there is essentially a common prior. The latter possibility is excluded by assumption. So we must have $\delta=0$, and therefore $\delta=\gamma$. Then, still by Equation (2), the two representations of $\succsim_{0}$ reduce to SEU representations: the one is given by $u_{0}$ and $\lambda_{0}$, the other one is given by $v_{0}$ and $\mu_{0}$. By the uniqueness part of the Anscombe-Aumann (1963) [3] theorem, we obtain $\mu_{0}=\lambda_{0}$, and $v_{0}$ is a positive affine transformation of $u_{0}$. However, the two utility functions are normalized. Hence $v_{0}=u_{0}$.

Suppose now $\gamma=1$. Then, by Equation (2), $\succsim_{0}$ must satisfy Pareto Dominance. But then, by Proposition 1, either $\delta=1$, or the social prior is essentially a common prior. The latter possibility is excluded by assumption. So we must have $\delta=1$, and therefore $\delta=\gamma$. Consider now the restriction of $\succsim_{0}$ to constant acts; that is, to lotteries in $Y$. By Theorem 1 , each of $u_{0}$ and $v_{0}$ provides a representation for this restriction. By the uniqueness part of the von Neumann and Morgenstern (1944) theorem, $u_{0}$ and $v_{0}$ must be positive affine transformation of each other. But since they are normalized, they are in fact equal to each other. Then, since $\gamma>0$ and $\delta>0$, we have $u_{0}=\sum_{i \in N} \alpha_{i} u_{i}$ and $v_{0}=\sum_{i \in N} \beta_{i} u_{i}$. Therefore, $\sum_{i \in N} \alpha_{i} u_{i}=\sum_{i \in N} \beta_{i} u_{i}$. Since Risk Diversity holds, we must have $\beta_{i}=\alpha_{i}$ for any $i \in N$ (Proceed as in Claim 1).

Finally, suppose $\gamma \in(0,1)$. Then, by the previous paragraphs, it must be the case that $\delta \in(0,1)$. Consider now the restriction of $\succsim_{0}$ to constant acts; that is, to lotteries in $Y$. By Theorem 1, each of $u_{0}$ and $v_{0}$ provides a representation for this restriction. By the uniqueness part of the von Neumann and Morgenstern (1944) theorem, $u_{0}$ and $v_{0}$ must be positive affine transformation of each other. But since they are normalized, they are in fact equal to each other. Let $V_{0}$ and $W_{0}$ be the functionals defined respectively by $\left\{\gamma,\left(\alpha_{i}\right)_{i \in N}, u_{0}, \lambda_{0}\right\}$ and $\left\{\delta,\left(\beta_{i}\right)_{i \in N}, v_{0}, \mu_{0}\right\}$ as in Equation (2). For any $l \in Y$, we have by Theorem $1 V_{0}(l)=u_{0}(l)=v_{0}(l)=W_{0}(l)$. Moreover, for each $f \in \mathcal{F}$, there exists $l \in Y$ such that $f \sim_{0} l$ (See proof of Theorem 1). Then, $V_{0}(f)=V_{0}(l)=W_{0}(l)=W_{0}(f)$. On the other hand, the functionals $V_{0}$ and $W_{0}$ can be written in the following way: for any $f \in \mathcal{F}$ 


$$
V_{0}(f)=\sum_{i \in N, s \in S}\left[\gamma \cdot \alpha_{i} \cdot \lambda_{i}(s)+(1-\gamma) \cdot \alpha_{i} \cdot \lambda_{0}(s)\right] \cdot u_{i}(f(s))
$$

and

$$
W_{0}(f)=\sum_{i \in N, s \in S}\left[\delta \cdot \beta_{i} \cdot \lambda_{i}(s)+(1-\delta) \cdot \beta_{i} \cdot \mu_{0}(s)\right] \cdot u_{i}(f(s)) .
$$

Then, define $\psi(i, s)=\left[\gamma \alpha_{i} \lambda_{i}(s)+(1-\gamma) \alpha_{i} \lambda_{0}(s)\right]-\left[\delta \beta_{i} \lambda_{i}(s)+(1-\delta) \beta_{i} \mu_{0}(s)\right]$ for any $i \in N$ and $s \in S$. By confronting Equations (31) and (32) and applying Lemma 3, we obtain:

$$
\gamma \cdot \alpha_{i} \cdot \lambda_{i}(s)+(1-\gamma) \cdot \alpha_{i} \cdot \lambda_{0}(s)=\delta \cdot \beta_{i} \cdot \lambda_{i}(s)+(1-\delta) \cdot \beta_{i} \cdot \mu_{0}(s) .
$$

By summing these equalities on $S$, we obtain $\beta_{i}=\alpha_{i}$ for any $i \in N$. Moreover, since there is essentially no common prior, there exist two non-null $i, j \in N$ such that $\lambda_{i} \neq \lambda_{j}$. Let $s \in S$ be such that $\lambda_{i}(s) \neq \lambda_{j}(s)$. Moreover, by Lemma 2, we have $\alpha_{i}>0$ and $\alpha_{j}>0$. By applying twice Equation (33) and substracting, we get

$$
\gamma \cdot\left(\lambda_{i}(s)-\lambda_{j}(s)\right)=\delta \cdot\left(\lambda_{i}(s)-\lambda_{j}(s)\right)
$$

Since $\lambda_{i}(s) \neq \lambda_{j}(s)$, it must be that $\delta=\gamma$. Finally, applying again Equation (33) to any $s \in S$ and some non-null individual $i \in N$ gives $\mu_{0}=\lambda_{0}$.

\subsection{Proof of Proposition 4}

Assume that $\succsim_{0}$ satisfies Weak Order, Continuity, Independence, Boundedness, Weak Dominance and Non-triviality. Then, by Theorem 1, there exists a structure $\left\{\gamma,\left(\alpha_{i}\right)_{i \in N}, u_{0}, \lambda_{0}\right\}$ providing a representation of $\succsim_{0}$ as in Theorem 1 . Let $V_{0}$ be the functional this structure defines according to Equation (2).

Moreover, let $I$ stand for the set of $i \in N$ that are non-null. Suppose momentarily that $\gamma>0$. Then, by Lemma $2, I=\left\{i \in N, \alpha_{i}>0\right\}$. If $i \in I$, then $\lambda_{i}=\lambda$. So for any $f \in \mathcal{F}$

$$
\begin{aligned}
V_{0}(f) & =\gamma \cdot \sum_{i \in I} \alpha_{i} \cdot \mathbb{E}_{\lambda}\left(u_{i} \circ f\right)+(1-\gamma) \cdot \mathbb{E}_{\lambda_{0}}\left(u_{0} \circ f\right) \\
& =\gamma \cdot \mathbb{E}_{\lambda}\left(\sum_{i \in I} \alpha_{i} \cdot u_{i} \circ f\right)+(1-\gamma) \cdot \mathbb{E}_{\lambda_{0}}\left(u_{0} \circ f\right) \\
& =\gamma \cdot \mathbb{E}_{\lambda}\left(u_{0} \circ f\right)+(1-\gamma) \cdot \mathbb{E}_{\lambda_{0}}\left(u_{0} \circ f\right)
\end{aligned}
$$

Note that the latter equality also holds if $\gamma=0$. So it holds for any $\gamma \in[0,1]$. This shows that $\succsim_{0}$ has a SEU representation where the normalized utility function is given by $u_{0}$ and the probability measure is given by $\gamma \lambda+(1-\gamma) \lambda_{0}$, thereby showing Equation (4) as well as (P4.1) and (P4.3). Moreover, (P4.2) follows from (Th1.1). Finally, the uniqueness of $\mu_{0}$ follows from the Anscombe and Aumann (1963) [3] theorem, which also provides the uniquenes of $v_{0}$ up to positive affine transformation. But since $v_{0}$ is normalized, it is in fact unique. 
Now, suppose that $\succsim_{0}$ has an SEU representation with respect to a normalized and mixture affine function $v_{0}: Y \rightarrow \mathbb{R}$ and probability measure $\mu_{0}$ on $S$. Then, it is easy to see that it satisfies all of Weak Order, Continuity, Independence, and Non-triviality. Moreover, $\succsim_{0}$ satisfies Statewise Dominance, and therefore satisfies Weak Dominance. Finally, since $\succsim_{0}$ satisfies Statewise Dominance, it also satisfies Boundedness.

\section{References}

[1] Akerlof, G. The market for lemons: Qualitative uncertainty and the market mechanism. Quarterly Journal of Economics 84 (1970).

[2] Alon, S., And Gayer, G. Utilitarian preferences with multiple priors. Econometrica 84, 3 (2016), 1181-1201.

[3] Anscombe, F. J., And Aumann, R. J. A definition of subjective probability. Annals of mathematical statistics (1963), 199-205.

[4] Billot, A., And Vergopoulos, V. Aggregation of paretian preferences for independent individual uncertainties. Social Choice and Welfare 47, 4 (2016), 973-984.

[5] Brunnermeier, M. K., Simsek, A., And Xiong, W. A welfare criterion for models with biased beliefs. Quarterly Journal of Economics 129, 4 (2014), 1711-1752.

[6] Chambers, C. P., And Hayashi, T. Preference aggregation under uncertainty: Savage vs. pareto. Games and Economic Behavior 54, 2 (2006), 430-440.

[7] Chambers, C. P., And Hayashi, T. Preference aggregation with incomplete information. Econometrica 82, 2 (2014), 589-599.

[8] Danan, E., Gajdos, T., Hill, B., and Tallon, J.-M. Robust social decisions. American Economic Review 106, 9 (September 2016), 2407-25.

[9] De Meyer, B., And Mongin, P. A note on affine aggregation. Economics Letters 47, 2 (1995), 177-183.

[10] Diamond, P. A., ET AL. Cardinal welfare, individualistic ethics, and interpersonal comparison of utility: Comment. The Journal of Political Economy 75, 5 (1967), 765.

[11] Dietrich, F., And List, C. Probabilistic opinion pooling. Oxford Handbook of Probability and Philosophy (2016).

[12] Fishburn, P. C. Utility theory for decision making. John Wiley and Sons, 1970.

[13] GilboA, I., Samet, D., And Schmeidler, D. Utilitarian aggregation of beliefs and tastes. Journal of Political Economy 112, 4 (2004), 932-938. 
[14] Gilboa, I., Samuelson, L., And Schmeidler, D. No-betting-pareto dominance. Econometrica 82, 4 (2014), 1405-1442.

[15] Harsanyi, J. Cardinal welfare, individualistic ethics, and interpersonal comparisons of welfare. Journal of Political Economy 63 (1955).

[16] Hausman, D., And McPherson, M. Preference, belief, and welfare. The American Economic Review 84, 2 (1994), 396-400.

[17] Hylland, A., And Zeckhauser, R. The impossibility of bayesian group decision making with separate aggregation of beliefs and values. Econometrica 47 (1979), 1321-1336.

[18] Kreps, D. Notes on the Theory of Choice. Westview Press: Underground Classics in Economics, 1988.

[19] Mongin, P. Consistent bayesian aggregation. Journal of Economic Theory 66, 2 (1995), 313-351.

[20] Mongin, P. Spurious unanimity and the pareto principle. Economics and Philosophy (1997), 1-22.

[21] Mongin, P. The paradox of the bayesian experts and state-dependent utility theory. Journal of Mathematical Economics 29, 3 (1998), 331-361.

[22] Mongin, P., And Pivato, M. Ranking multidimensional alternatives and uncertain prospects. Journal of Economic Theory 157 (2015), 146-171.

[23] Qu, X. Separate aggregation of beliefs and values under ambiguity. Economic Theory (2014), 1-17.

[24] Savage, L. J. The foundations of statistics. 